TRANSACTIONS OF THE

AMERICAN MATHEMATICAL SOCIETY

Volume 350, Number 11, November 1998, Pages 4313-4339

S $0002-9947(98) 02353-8$

\title{
PROJECTIVITY, TRANSITIVITY AND AF-TELESCOPES
}

\author{
TERRY A. LORING AND GERT K. PEDERSEN
}

\begin{abstract}
Continuing our study of projective $C^{*}$-algebras, we establish a projective transitivity theorem generalizing the classical Glimm-Kadison result. This leads to a short proof of Glimm's theorem that every $C^{*}$-algebra not of type I contains a $C^{*}$-subalgebra which has the Fermion algebra as a quotient. Moreover, we are able to identify this subalgebra as a generalized mapping telescope over the Fermion algebra.

We next prove what we call the multiplier realization theorem. This is a technical result, relating projective subalgebras of a multiplier algebra $M(A)$ to subalgebras of $M(E)$, whenever $A$ is a $C^{*}$-subalgebra of the corona algebra $C(E)=M(E) / E$. We developed this to obtain a closure theorem for projective $C^{*}$-algebras, but it has other consequences, one of which is that if $A$ is an extension of an MF (matricial field) algebra (in the sense of Blackadar and Kirchberg) by a projective $C^{*}$-algebra, then $A$ is MF.

The last part of the paper contains a proof of the projectivity of the mapping telescope over any $\mathrm{AF}$ (inductive limit of finite-dimensional) $C^{*}$-algebra. Translated to generators, this says that in some cases it is possible to lift an infinite sequence of elements, satisfying infinitely many relations, from a quotient of any $C^{*}$-algebra.
\end{abstract}

\section{INTRODUCTION}

Recall from [17] that a $C^{*}$-algebra $P$ is projective, if for every pair of $C^{*}$-algebras $B, C$ such that $\pi: B \rightarrow C$ is a surjective morphism (throughout the paper morphism means ${ }^{*}$-homomorphism), and for each morphism $\varphi: P \rightarrow C$, there is a morphism $\psi: P \rightarrow B$ such that $\pi \circ \psi=\varphi$. In diagrammatic notation:

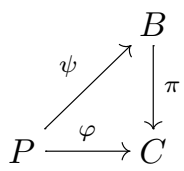

This definition and the basic properties of projective $C^{*}$-algebras are due to Effros and Kaminker [9]. There is also a definition of a projective morphism due to Blackadar [3]. It was proved in $[18,2.2]$ (and we shall use this fact repeatedly) that it suffices to show that morphisms lift from corona algebras to multiplier algebras.

Received by the editors November 7, 1994.

1991 Mathematics Subject Classification. Primary 46L05.

Key words and phrases. Projectivity, transitivity, multipliers, telescopes, Bratteli diagram, Glimm's theorem, MF algebra.

This research was made possible through a NATO Collaboration Grant (\# 920177). Both authors also acknowledge the support of their respective science foundations: NFS (\# DMS9215024) and SNF; and the second author recalls with gratitude the hospitality offered (twice!) by the Department of Mathematics at the University of New Mexico.

(C) 1998 American Mathematical Society 
Thus, in the separable case, $P$ is projective if for every $\sigma$-unital $C^{*}$-algebra $A$ with multiplier algebra $M(A)$ and corona algebra $C(A)=M(A) / A$, we can solve the lifting problem

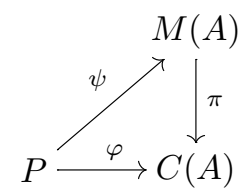

Evidently projective $C^{*}$-algebras must be rather special: If $\mathbf{C} P$ denotes the mapping cone over $P$, i.e.

$$
\left.\left.\left.\left.\mathbf{C} P=C_{0}(] 0,1\right]\right) \otimes P=C_{0}(] 0,1\right], P\right),
$$

then the map $f \rightarrow f(1)$ is a surjection of $\mathbf{C} P$ onto $P$. Therefore, if $P$ is projective, it has an embedding as a $C^{*}$-subalgebra of $\mathbf{C} P$. Now $\mathbf{C} P$ is contractible - in any conceivable sense - and thus so is $P$. In particular, $K_{0}(P)=K_{1}(P)=0$. Moreover, since $\mathbf{C} P$ contains no non-zero projections, $P$ is never unital.

Another peculiar property of a projective $C^{*}$-algebra $P$ is that it must have a separating family of finite-dimensional representations (see [14]), i.e. $P$ must be residually finite-dimensional. (See [10] for equivalent formulations of this condition.) To show this, at least in the separable case, write the separable Hilbert space $\mathfrak{H}$ as the inductive limit of $n$-dimensional subspaces $\mathfrak{H}_{n}, n \in \mathbb{N}$, and in the direct product $C^{*}$-algebra of matrix algebras $\prod \mathbb{B}\left(\mathfrak{H}_{n}\right)$ take the $C^{*}$-subalgebra $A$ of all strong* convergent sequences (relative to the embeddings $\mathfrak{H}_{n} \rightarrow \mathfrak{H}_{n+1}$ ) and the closed ideal $I$ of $A$ consisting of sequences converging to zero. Then $A / I=\mathbb{B}(\mathfrak{H})$. Evidently $P$ has a faithful representation $\varphi: P \rightarrow \mathbb{B}(\mathfrak{H})$, thus, being projective, also an embedding as a $C^{*}$-subalgebra of $A$, which is clearly residually finite-dimensional.

It is the purpose of this paper to show that - despite the above-mentioned restrictions - there is a rich and fascinating supply of projective $C^{*}$-algebras. Indeed, every mapping telescope of an AF-algebra is projective by Theorem 7.2. The strategic importance of projective algebras lies in the fact that they provide an algebraic setting of lifting problems, which otherwise have a tendency to degenerate into single operator theory.

A major detour through the structure of corona algebras is unavoidable on the way to our goal of proving various $C^{*}$-algebras to be projective. This is not a bad thing, as the focus moves from representation theory, with $\mathfrak{K}(\mathfrak{H}), \mathfrak{B}(\mathfrak{H}), \mathfrak{Q}(\mathfrak{H})$ playing the starring roles, to almost multiplicative maps and asymptotic morphisms, where corona algebras such as $\prod M_{n} / \bigoplus M_{n}$ and $C_{b}\left(\left[1, \infty[, B) / C_{0}([1, \infty[, B)\right.\right.$ (assuming $B$ unital) are prominent. We will discuss connections with asymptotic morphisms, and so with the Connes-Higson $E$-theory, in a future paper, but do include here a result that gives some evidence for a conjecture of Blackadar and Kirchberg $[3, \S 7]$ regarding $M F$ algebras.

\section{Three Items to Recall}

Universal $C^{*}$-algebras. Given a set $G$ of generators, and a set $R$ of relations between elements in $G$, there is a universal algebra $\langle G \mid R\rangle$ generated by $G$ satisfying $R$. If the relations $R$ contain or imply norm restrictions on the generators, there is a universal $C^{*}$-algebra $C^{*}\langle G \mid R\rangle$, which surjects onto any other $C^{*}$-algebra $A$ that 
is generated (as a $C^{*}$-algebra) by elements $\left\{a_{g} \in A \mid g \in G\right\}$ satisfying the relations $R$. Often enough, universal $C^{*}$-algebras are hopelessly complicated. Consider e.g.

$$
C^{*}\langle x \mid\|x\| \leq 1\rangle
$$

which, although it is projective, will surject onto any singly generated $C^{*}$-algebra. Sometimes they are quite harmless though. Thus

$$
C^{*}\left\langle x \mid\|x\| \leq 1, x^{2}=0\right\rangle \cong \mathbb{C M}_{2},
$$

cf. $[16,1.4]$. Note that we have defined universality in the category of non-unital (i.e. not necessarily unital) $C^{*}$-algebras. Note also that to define a morphism $\pi$ from a universal $C^{*}$-algebra $C^{*}\langle G \mid R\rangle$ into a $C^{*}$-algebra $A$, it suffices to define $\pi(g)$ in $A$ for each $g$ in $G$, and then check that the $\pi(g)$ 's satisfy the relations $R$.

Split Extensions. As usual we say that a $C^{*}$-algebra $A$ is an extension of $P$ by $Q$, if there is a short exact sequence

$$
0 \rightarrow P \stackrel{\iota}{\rightarrow} A \stackrel{\pi}{\rightarrow} Q \rightarrow 0
$$

As shown by Busby ([5], see also [25, Ch. 3]), extensions are classified by the set of morphisms from $Q$ into the corona algebra $M(P) / P$. In this paper we shall mostly deal with extensions where the quotient $Q$ is projective, in which case, of course, the extension splits, i.e. there is an injective morphism $\lambda: Q \rightarrow A$, such that $\pi \circ \lambda=$ id. For easy reference we note the following well-known result:

2.1. Proposition. There is a bijective correspondence between split extensions (with specified splitting) $A$ of $P$ by $Q$, and morphisms $\theta: Q \rightarrow M(P)$, given in one direction by

$$
A=\{(q, m) \in Q \oplus M(P) \mid \theta(q)-m \in P\},
$$

where $\iota(p)=(0, p), \pi(q, m)=q$ and $\lambda(q)=(q, \theta(q))$; and in the other by setting

$$
\theta(q) p=\lambda(q) \iota(p) \quad(q \in Q, p \in P) .
$$

Proof. Straightforward computations.

2.2. Proposition. If $A$ is a split extension of $P$ by $Q$ given by the morphism $\theta: Q \rightarrow M(P)$, and $B$ is another $C^{*}$-algebra, there is a bijective correspondence between morphisms $\pi: A \rightarrow B$ and pairs $(\varphi, \psi)$ of morphisms $\varphi: P \rightarrow B$ and $\psi: Q \rightarrow B$ satisfying

$$
\varphi(p \theta(q))=\varphi(p) \psi(q) \quad(p \in P, q \in Q) .
$$

Proof. Since $A$ is the universal $C^{*}$-algebra with generators $P \cup Q$ and relations $p \cdot q=p \theta(q), p \in P, q \in Q$, this follows from the remark above.

Telescope Algebras. Let $A=\overline{\bigcup A_{n}}$ be an inductive limit of a sequence of $C^{*}$ algebras $\left(A_{n}\right)$, where the embeddings $A_{1} \hookrightarrow A_{2} \hookrightarrow \ldots$ simply are regarded as inclusion maps. If all the algebras $A_{n}$ are unital and the embeddings are unitpreserving, we talk about a unital inductive limit. Following Brown (see $[24,5.2]$ ), we define the mapping telescope on $\left(A_{n}\right)$ as the $C^{*}$-algebra

$$
\left.\left.\mathbf{T}(A)=\left\{f \in C_{0}(] 0, \infty\right], A\right) \mid t \leq n \Rightarrow f(t) \in A_{n}\right\} .
$$

If we let $\mathbf{T}\left(A_{1}, A_{2}, \ldots, A_{n}\right)$ equal

$$
\left\{\begin{array}{l|l}
\left.\left.f \in C_{0}(] 0, \infty\right], A_{n}\right) & \begin{array}{c}
t \leq k \Rightarrow f(t) \in A_{k} \text { and } \\
t \geq n \Rightarrow f(t)=f(n)
\end{array}
\end{array}\right\},
$$


then we have embeddings

$$
\mathbf{T}\left(A_{1}\right) \subset \mathbf{T}\left(A_{1}, A_{2}\right) \subset \cdots \subset \mathbf{T}(A),
$$

and it is easily verified that the infinite telescope $\mathbf{T}(A)$ is the inductive limit of the finite telescopes $\mathbf{T}\left(A_{1}, \ldots, A_{n}\right)$. The relevance of telescopes for ${ }^{*}$-algebras should be obvious - even to the layman.

2.3. Remark. Clearly the telescope $C^{*}$-algebra $\mathbf{T}(A)$ depends not only on $A$, but also on the sequence $\left(A_{n}\right)$, so that the notation is highly ambiguous. In the interest of brevity we shall nevertheless retain the compact symbol $\mathbf{T}(A)$ instead of the more correct $\mathbf{T}\left(A_{1}, A_{2}, \ldots\right)$. For the finite telescopes, however, the longer designation $\mathbf{T}\left(A_{1}, \ldots, A_{n}\right)$ will be necessary.

If for each $n$ we identify $] 0,1]$ with $] n-1, n]$, there is a natural embedding of the cone $\mathbf{C} A_{n}$ as a closed ideal of the telescope algebra $\mathbf{T}\left(A_{1}, \ldots, A_{n}\right)$, where

$$
\mathbf{C} A_{n}=\left\{f \in \mathbf{T}\left(A_{1}, \ldots, A_{n}\right) \mid t \leq n-1 \Rightarrow f(t)=0\right\} .
$$

Assuming that we have a unital inductive limit, this leads to the following.

2.4. Proposition. Each finite telescope $\mathbf{T}\left(A_{1}, \ldots, A_{n}\right)$ is the split extension of $\mathbf{C} A_{n}$ by $\mathbf{T}\left(A_{1}, \ldots, A_{n-1}\right)$ determined by the morphism $\theta$ of $\mathbf{T}\left(A_{1}, \ldots, A_{n-1}\right)$ into $\left.\left.\left.M\left(\mathbf{C} A_{n}\right)\left(=C_{b}(] 0,1\right]\right), A_{n}\right)\right)$ given by $\theta f(t)=f(n-1)$ for $\left.\left.t \in\right] 0,1\right]$.

Proof. Recall from Proposition 2.1 that the split extension $B$ determined by $\theta$ is

$$
\left.\left.\left.B=\left\{(f, m) \in \mathbf{T}\left(A_{1}, \ldots, A_{n-1}\right) \oplus C_{b}(] 0,1\right]\right), A_{n}\right) \mid m-\theta(f) \in \mathbf{C} A_{n}\right\} .
$$

Since $\theta f$ is a constant map with value in $A_{n-1}$, this means that $m$ must be continuous on $[0,1]$ with $m(0)=f(n-1)$. Define $\gamma: B \rightarrow \mathbf{T}\left(A_{1}, \ldots, A_{n}\right)$ by

$$
\gamma(f, m)(t)= \begin{cases}f(t), & 0<t \leq n-1, \\ m(t+1-n), & n-1 \leq t \leq n, \\ m(t), & n \leq t .\end{cases}
$$

Elementary, albeit somewhat lengthy computations show that $\gamma$ is a *-isomorphism of $B$ onto $\mathbf{T}\left(A_{1}, \ldots, A_{n}\right)$. Its inverse is given by restriction: $\gamma^{-1}(f)=(r f, m)$, where

$$
r f(t)= \begin{cases}f(t), & 0<t \leq n-1 \\ f(n-1), & n-1 \leq t\end{cases}
$$

and

$$
m(t)=f(n-1+t)-f(n-1) .
$$

Combining Propositions 2.2 and 2.4 we have

2.5. Corollary. Given a unital inductive limit $A=\overline{\bigcup A_{n}}$ and a sequence of morphisms $\varphi_{n}: \mathbf{C} A_{n} \rightarrow B$ into some $C^{*}$-algebra $B$, satisfying

$$
\varphi_{m}(f) \varphi_{n}(g)=\varphi_{n}(f(1) g) \quad(m<n),
$$

there is a morphism $\varphi: \mathbf{T}(A) \rightarrow B$ such that $\varphi_{\mid \mathbf{C} A_{n}}=\varphi_{n}$ for all $n$, where each cone $\mathbf{C} A_{n}$ is regarded as an ideal in $\mathbf{T}\left(A_{1}, \ldots, A_{n}\right)$. 


\section{Preliminary Lifting Results}

We have shown previously, [16, 4.2], that the mapping cone over a finite-dimensional $C^{*}$-algebra is projective. We shall need a slightly stronger result in which some elements are constrained to be less than some given orthogonal elements "upstairs". Contained in this is a shorter proof of the projectivity of $\mathbf{C M}_{n}$.

If not otherwise specified, $A$ will denote a $C^{*}$-algebra, $I$ a closed ideal and $\pi: A \rightarrow A / I$ the quotient map.

3.1. Lemma. In any $C^{*}$-algebra, if $a^{*} a \leq b^{*} b$ and $c c^{*} \leq d d^{*}$, then for each $x$,

$$
\|a x c\| \leq\|b x d\| .
$$

Proof. We have

$$
\begin{aligned}
\|a x c\|^{2} & =\left\|c^{*} x^{*} a^{*} a x c\right\| \leq\left\|c^{*} x^{*} b^{*} b x c\right\| \\
& =\left\|b x c c^{*} x^{*} b^{*}\right\| \leq\left\|b x d d^{*} x^{*} b^{*}\right\|=\|b x d\|^{2} .
\end{aligned}
$$

3.2. Lemma. Suppose that $a, b$ and $c$ are elements in $a C^{*}$-algebra, such that $a^{*} a \leq b$ and $c c^{*} \leq b$. Then the limit

$$
x=\lim a\left(\frac{1}{n} 1+b\right)^{-1 / 2} c
$$

exists (in norm). Moreover:

(i) $x^{*} x \leq c^{*} c$;

(ii) $x x^{*} \leq a a^{*}$;

(iii) if $c c^{*}=b$ then $x x^{*}=a a^{*}$;

(iv) if $c=b^{1 / 2}$ then $x=a$.

Proof. See [21, 1.1.4 and 1.1.5], or use the previous lemma plus functional calculus.

The following two-sided version of Combes' order lifting theorem (cf. [21, 1.5.10]) was proved by Davidson $[8,2.4]$. Here is a short proof.

3.3. Theorem. Suppose that $a$ and $b$ are positive elements in $A$ and $y \in A / I$ such that $y^{*} y \leq \pi(a)$ and $y y^{*} \leq \pi(b)$. Then there is a lift $x$ in $A$ of $y$ with $x^{*} x \leq a$ and $x x^{*} \leq b$.

Proof. Let $z$ be any lift of $y$ and put $c=\left(z^{*} z-a\right)_{+}+a$, so that $z^{*} z \leq c, a \leq c$, and $\pi(c)=\pi(a)$. By Lemma 3.2 (i) and (iv) we have $x_{0}=\lim z\left(\frac{1}{n} 1+c\right)^{-1 / 2} a^{1 / 2}$ in $A$ with $x_{0}^{*} x_{0} \leq a$ and $\pi\left(x_{0}\right)=y$. Now put $d=\left(x_{0} x_{0}^{*}-b\right)_{+}+b$, so that $x_{0} x_{0}^{*} \leq d, b \leq d$, and $\pi(d)=\pi(b)$. By Lemma $3.2(\mathrm{i})$, (ii) and (iv) we have $x=\lim b^{1 / 2}\left(\frac{1}{n} 1+d\right)^{-1 / 2} x_{0}$ in $A$ with $x x^{*} \leq b, x^{*} x \leq x_{0}^{*} x_{0} \leq a$, and $\pi(x)=y$, as desired.

3.4. Proposition. Suppose that $k_{1}, \ldots, k_{n}$ are mutually orthogonal, positive elements in $A / I$ of norm at most one. Then there are elements $h_{1}, h_{2}, \ldots, h_{n}$ in $A$ with the same properties, such that $\pi\left(h_{j}\right)=k_{j}$ for all $j$.

Proof. Put $b=\sum_{j=2}^{n} 2^{-j} k_{j}$, and let $x$ be a self-adjoint element in $A$ with $\pi(x)=$ $k_{1}-b$. Define $f(t)=(t \vee 0) \wedge 1$ and $g(t)=-(t \wedge 0)$, and put $h_{1}=f(x)$. Then $\pi\left(h_{1}\right)=f\left(k_{1}-b\right)=k_{1}$, and if $A_{1}$ denotes the two-sided annihilator of $h_{1}$, then 
$k_{2}, \ldots, k_{n}$ belong to $\pi\left(A_{1}\right)$, since $g(x) \in A_{1}$ and $\pi(g(x))=b$. The argument now proceeds by induction. As can be seen from $[19,6.5]$, the argument can be used to lift a whole sequence of orthogonal elements.

3.5. Theorem. Suppose that $\varphi: \mathbb{C M}_{n} \rightarrow A / I$ is a morphism of the mapping cone over some $\mathbb{M}_{n}$, and suppose we have chosen mutually orthogonal elements $h_{1}, \ldots, h_{n}$ in $A$ with $0 \leq h_{j} \leq 1$ and $\pi\left(h_{j}\right)=\varphi\left(\operatorname{id} \otimes e_{j j}\right)$ for $1 \leq j \leq n$. Then there is a morphism $\psi: \mathbf{C M}_{n} \rightarrow A$ with $\pi \circ \psi=\varphi$, such that $\psi\left(\mathrm{id} \otimes e_{j j}\right) \leq h_{j}$ for all $j$.

Proof. Recall from $[16,2.7]$ that $\mathbf{C M}_{n}$ is the universal $C^{*}$-algebra generated by the contractions $a_{j}\left(=\mathrm{id} \otimes e_{j 1}\right), 2 \leq j \leq n$, subject to the relations

$$
\begin{aligned}
\left\|a_{j}\right\| & \leq 1, & & \text { for all } j, \\
a_{j}^{*} a_{k} & =0, & & \text { if } j \neq k, \\
a_{j}^{*} a_{j} & =a_{k}^{*} a_{k}, & & \text { for all } j, k, \\
a_{j}^{2} & =0 & & \text { for all } j .
\end{aligned}
$$

Applying Theorem 3.3 we find a lift $y_{n}$ in $A$ of the element $\varphi\left(a_{n}\right)$ such that

$$
y_{n}^{*} y_{n} \leq h_{1}^{2}, \quad y_{n} y_{n}^{*} \leq h_{n}^{2}
$$

Applying it again we find a lift $y_{n-1}$ of $\varphi\left(a_{n-1}\right)$ satisfying

$$
y_{n-1}^{*} y_{n-1} \leq y_{n}^{*} y_{n}, \quad y_{n-1} y_{n-1}^{*} \leq h_{n-1}^{2} \text {. }
$$

Continuing by induction, we end up with elements $y_{2}, y_{3}, \ldots, y_{n}$ in $A$ such that

$$
\begin{aligned}
\pi\left(y_{j}\right) & =\varphi\left(a_{j}\right), \\
y_{j} y_{j}^{*} & \leq h_{j}^{2} \\
y_{2}^{*} y_{2} & \leq \cdots \leq y_{n}^{*} y_{n} \leq h_{1}^{2} .
\end{aligned} \quad(2 \leq j \leq n),
$$

Except for the penultimate condition, these elements satisfy the relations $(*)$. We correct them by setting

$$
x_{j}=\lim y_{j}\left(\frac{1}{n} 1+y_{j}^{*} y_{j}\right)^{-1 / 2}\left(y_{2}^{*} y_{2}\right)^{1 / 2},
$$

for $2 \leq j \leq n$, which exist in $A$ by Lemma 3.2 and satisfy the relations $(*)$. By universality (cf. Section 1) there is therefore a morphism $\psi: \mathbf{C M}_{n} \rightarrow A$ given by $\psi\left(a_{j}\right)=x_{j}, 2 \leq j \leq n$. Since $\pi\left(x_{j}\right)=\varphi\left(a_{j}\right)$, and the $a_{j}$ 's are generators, it follows that $\pi \circ \psi=\varphi$, and clearly

$$
\psi\left(\mathrm{id} \otimes e_{j j}\right)=\psi\left(\left(a_{j} a_{j}^{*}\right)^{1 / 2}\right)=\left(x_{j} x_{j}^{*}\right)^{1 / 2} \leq\left(y_{j} y_{j}^{*}\right)^{1 / 2} \leq h_{j},
$$

since the square root is operator monotone.

3.6. Corollary. Let $F$ be a finite-dimensional $C^{*}$-algebra and let $p_{1}, \ldots, p_{n}$ be a set of mutually orthogonal, one-dimensional projections in $F$, summing to the identity. Assume that $\varphi: \mathbf{C} F \rightarrow A / I$ is a morphism, and $h_{1}, \ldots, h_{n}$ is a set of mutually orthogonal elements in $A$ with $0 \leq h_{j} \leq 1$ and $\pi\left(h_{j}\right)=\varphi\left(\mathrm{id} \otimes p_{j}\right)$ for all $j$. Then there is a morphism $\psi: \mathbf{C} F \rightarrow A$ such that $\pi \circ \psi=\varphi$ and $\psi\left(\mathrm{id} \otimes p_{j}\right) \leq h_{j}$ for all $j$.

3.7. Theorem. Let $F$ be a finite-dimensional $C^{*}$-algebra and let $q_{1}, \ldots, q_{m}$ be an orthogonal family of projections (of any dimensions) summing to the identity in $F$. Given any morphism $\varphi: \mathbf{C} F \rightarrow A / I$ and mutually orthogonal hereditary $C^{*}$ subalgebras $A_{1}, \ldots, A_{m}$ of $A$ such that $\varphi\left(\mathrm{id} \otimes q_{j}\right) \in \pi\left(A_{j}\right)$ for $1 \leq j \leq m$, there is a morphism $\psi: \mathbf{C} F \rightarrow A$ such that $\pi \circ \psi=\varphi$ and $\psi\left(\mathrm{id} \otimes q_{j}\right) \in A_{j}$ for all $j$. 
Proof. Let $p_{1}, \ldots, p_{n}$ be an orthogonal family of one-dimensional projections in $F$, summing to the identity and subordinate to the $q_{j}$ 's. By Lemma 3.4 there are mutually orthogonal elements $h_{1}, \ldots, h_{n}$ in $A$ with $0 \leq h_{i} \leq 1$ and $\pi\left(h_{i}\right)=$ $\varphi\left(\right.$ id $\left.\otimes p_{i}\right)$ for all $i$, and such that $h_{i} \in A_{j}$ whenever $p_{i} \leq q_{j}$. Now apply Corollary 3.6 and the fact that the $A_{j}$ 's are hereditary.

3.8. Corollary. The mapping cone $\mathbf{C} F$ over any finite-dimensional $C^{*}$-algebra $F$ is projective.

A key consequence (see $[17,3.3]$ ) of the fact that $\mathbf{C M}_{n}$ is projective is that the class of ( $\sigma$-unital) projective $C^{*}$-algebras is closed under tensoring with matrix algebras. Similarly, the fact that the cone over $\mathbb{C} \oplus \mathbb{C}$ is projective has as a consequence that the $(\sigma$-unital) projectives are stable under direct sums. The more elementary fact that if $A$ and $B$ are $C^{*}$-algebras and $\widetilde{A}=\widetilde{B}$, then both or none of $A$ and $B$ are projective, is verified directly.

These closure properties were used in [18] to show that $C_{0}(X) \otimes \mathbb{M}_{n}$ is projective whenever $X$ is a finite tree. We can now handle more general subhomogeneous $C^{*}$-algebras over finite trees. While this will follow from more general results later, we give an example to show how the more precise lifting results involving $\mathbf{C M}_{n}$ are useful.

3.9. Example. Let $B$ denote the universal $C^{*}$-algebra generated by $h_{1}, \ldots, h_{n}$ and $a_{2}, \ldots, a_{n}$, subject to the relations

$$
\begin{aligned}
0 & \leq h_{j} \leq 1, & & \text { for all } j, \\
\left\|a_{j}\right\| & \leq 1, & & \text { for all } j, \\
h_{j} h_{k} & =0, & & \text { if } j \neq k, \\
a_{j}^{*} a_{j} & =a_{k}^{*} a_{k}, & & \text { for all } j, k, \\
a_{j} h_{1} & =h_{j} a_{j}=a_{j}, & & \text { for all } j .
\end{aligned}
$$

It is easily seen that there is a surjection

$$
\left.\left.\varphi: B \rightarrow\left\{f \in C_{0}(] 0,2\right], \mathbb{M}_{n}\right) \mid f(t) \text { is diagonal if } t \leq 1\right\}
$$

such that

$$
\varphi\left(h_{j}\right)=r \otimes e_{j j}, \quad \varphi\left(a_{j}\right)=s \otimes e_{j 1},
$$

where $r(t)=t \wedge 1$ and $s(t)=(t-1) \vee 0$. In fact $\varphi$ is an isomorphism.

To prove injectivity (see $[18,4.3]$ ) assume - for ease of notation - that $h_{1}, \ldots, h_{n}$ and $a_{2}, \ldots, a_{n}$ are operators on a Hilbert space $\mathfrak{H}$ (still satisfying the relations, of course) and generate an irreducible $C^{*}$-algebra. The element $\sum h_{j}$ is central, and thus $\sum h_{j}=\alpha 1$ for some scalar $\alpha$ with $0 \leq \alpha \leq 1$. But since $\sum h_{j}$ acts as a unit against all the $a_{j}$ 's we must have $\alpha \neq 0$. There are three cases to consider.

If $\alpha<1$, the relation $a_{j} h_{j}=a_{j}$ implies that $a_{j}=0$ for all $j$. The only time the orthogonal elements $h_{j}$ can act irreducibly is when $\operatorname{dim} \mathfrak{H}=1$, so $h_{j}=\alpha$ for some $j$ and $h_{k}=0$ for $k \neq j$. The representation is therefore the pull-back of $\varphi$ of a subrepresentation of evaluation at $\alpha$.

If $\alpha=1$ the element below is central, so for some scalar $\beta$ in $[0,1]$ we have

$$
a_{2}^{*} a_{2}+\sum_{j=2}^{n} a_{j} a_{j}^{*}=\beta 1
$$


When $\beta=0$ we have $a_{j}=0$ for $2 \leq j \leq n$, and we proceed as in the first case to show that the representation is the pull-back of $\varphi$ of a subrepresentation of evaluation at 1 .

In the third case $\alpha=1$ and $0<\beta \leq 1$. Then we have two sets of mutually orthogonal projections, $h_{1}, \ldots, h_{n}$, and $\beta^{-1} a_{2}^{*} a_{2}, \beta^{-1} a_{2} a_{2}^{*}, \ldots \beta^{-1} a_{n} a_{n}^{*}$, both summing to 1 . Since $a_{2}^{*} a_{2} \leq h_{1}$ and $a_{j} a_{j}^{*} \leq h_{j}$ for $2 \leq j \leq n$, this forces $a_{2}^{*} a_{2}=\beta h_{1}$ and $a_{j} a_{j}^{*}=\beta h_{j}$ for $2 \leq j \leq n$. Therefore $C^{*}\left(a_{2}, \ldots, a_{n}\right)^{\prime}=\mathbb{C} 1$, and we have $a_{j} a_{k}=0$ and, for $j \neq k, a_{j}^{*} a_{k}=0$, whereas $a_{j}^{*} a_{j}=a_{k}^{*} a_{k}$. But this is an irreducible representation of the cone $\mathbf{C M}_{n}$, and thus the pull-back of $\varphi$ of evaluation at $t=1+\beta^{1 / 2}$.

We have shown that all irreducible representations of the universal $C^{*}$-algebra $B$ are pull-backs of $\varphi$, which proves that $\varphi$ is an isomorphism.

3.10. Proposition. The $C^{*}$-algebra $B=\mathbf{T}\left(\mathbb{C}^{n}, \mathbb{M}_{n}\right)$, that is

$$
\left.\left.B=\left\{f \in C_{0}(] 0,2\right], \mathbb{M}_{n}\right) \mid t \leq 1 \Rightarrow f(t) \text { is diagonal }\right\}
$$

is projective.

Proof. Let $Q$ denote the $C^{*}$-subalgebra of $B$ consisting of diagonal functions on ]0,2] which are constant on $[1,2]$, and let $P$ denote the closed ideal of functions vanishing on ]0,1]. Then $P$ is isomorphic to $\mathbf{C M}_{n}$ (identifying ]0,1] with ]1,2]) and $B$ is the split extension of $P$ by $Q$.

Given a morphism $\varphi: B \rightarrow A / I$, we can find a lift $\psi_{2}: Q \rightarrow A$ of $\varphi \mid Q$, because $Q$, being isomorphic to $\mathbf{C} \mathbb{C}^{n}$, is projective. Let $r(t)=t \wedge 1$ and $s(t)=(1-t) \vee 0$ as before, and put $h_{j}=\psi_{2}\left(r \otimes e_{j j}\right), 1 \leq j \leq n$, so that $h_{1}, \ldots, h_{n}$ is a set of mutually orthogonal, positive contractions in $A$. Define

$$
A_{j}=\left\{x \in A \mid h_{j} x=x h_{j}=x\right\} \quad(1 \leq j \leq n)
$$

to obtain mutually orthogonal hereditary $C^{*}$-subalgebras of $A$, and note that $\varphi\left(s \otimes e_{j j}\right) \in \pi\left(A_{j}\right)$ for all $j$. By Theorem 3.7 there is a lift $\psi_{1}: P \rightarrow A$ of $\left.\varphi\right|_{P}$ such that $\psi_{1}\left(s \otimes e_{j j}\right) \in A_{j}$ for all $j$. With $\delta$ the Kronecker symbol this implies that

$$
\psi_{1}\left(s \otimes e_{j j}\right) \psi_{2}\left(r \otimes e_{i i}\right)=\psi_{1}\left(s \otimes e_{j j}\right) \delta_{i j}
$$

whence

$$
\psi_{1}\left(s \otimes e_{j j}\right) \psi_{2}\left(f \otimes e_{i i}\right)=\psi_{1}\left(s \otimes e_{j j}\right) f(1) \delta_{i j}
$$

for every $f$ in $\left.\left.C_{0}(] 0,2\right]\right)$, constant on $[1,2]$. In the general case, where $f=\sum f_{j} \otimes e_{j j}$ is in $Q$ and $g=\sum g_{i j} \otimes e_{i j}$ is in $P$, we get

$$
\begin{aligned}
\psi_{1}(g) \psi_{2}(f) & =\sum \psi_{1}\left(g_{i j} \otimes e_{i j}\right) \psi_{2}\left(f_{j} \otimes e_{j j}\right) \\
& =\sum \psi_{1}\left(g_{i j} \otimes e_{i j}\right) f_{j}(1) \\
& =\psi_{1}(g f(1)) .
\end{aligned}
$$

By Corollary 2.5 the pair $\left(\psi_{1}, \psi_{2}\right)$ defines a morphism $\psi: B \rightarrow A$, which is clearly a lift of $\varphi$, since $B=P+Q$.

3.11. Example. Let $B$ denote the universal $C^{*}$-algebra generated by contractions $x_{1}, \ldots, x_{n}$ satisfying the relations

$$
\begin{array}{ll}
x_{i}^{*} x_{i}=x_{j}^{*} x_{j}, & \text { for all } i, j, \\
x_{i}^{*} x_{j}=0, & \text { if } i \neq j .
\end{array}
$$


If we add the relations $x_{1} \geq 0$ (whence $x_{1}=\left(x_{j}^{*} x_{j}\right)^{1 / 2}$ for $2 \leq j \leq n$ ) and $x_{i} x_{j}=0$ if $i \neq j$, we have the relations for $\mathbf{C M}_{n}$; cf. (*) in the proof of Theorem 3.5. This means that there is a surjective morphism of $B$ onto $\mathbf{C M}_{n}$. If, on the other hand, we add the relations $x_{i}^{*} x_{i}=1,1 \leq i \leq n$ (or just $x_{1}^{*} x_{1}=1$ ), we have the relations for Cuntz's $C^{*}$-algebra $O_{n}$ (cf. [6]). There is therefore also a surjective morphism of $B$ onto $O_{n}$. Now the surprise:

3.12. Proposition. The $C^{*}$-algebra $B$ defined above is projective.

Proof. Given a morphism $\varphi: B \rightarrow A / I$, we can find, using Proposition 3.4, orthogonal, positive contractions $h_{1}, \ldots, h_{n}$ in $A$, such that $\pi\left(h_{j}\right)=\varphi\left(\left(x_{j} x_{j}^{*}\right)^{1 / 2}\right)$. Applying Theorem 3.3 recursively, we find that there are elements $y_{1}, \ldots, y_{n}$ in $A$ with $\pi\left(y_{i}\right)=x_{j}$, such that $y_{j} y_{j}^{*} \leq h_{j}^{2}$ and $y_{j+1}^{*} y_{j+1} \leq y_{j}^{*} y_{j}$ for all $j$. The renormalization

$$
z_{j}=\lim y_{j}\left(\frac{1}{n} 1+y_{j}^{*} y_{j}\right)^{-1 / 2}\left(y_{n}^{*} y_{n}\right)^{1 / 2}
$$

which exists in $A$ by Lemma 3.2 , produces elements $z_{1}, \ldots, z_{n}$ in $A$ that satisfy conditions $(*)$ in Example 3.11, and thus we have a morphism $\psi: B \rightarrow A$. That $\pi \circ \psi=\varphi$ follows from the fact that $\pi\left(z_{j}\right)=\varphi\left(x_{j}\right)$ for all $j$. This, in turn, follows from (iv) in Lemma 3.2.

\section{Transitivity Theorems}

Recall the following version of the Glimm-Kadison transitivity theorem (cf. [13] or $[21,2.7 .5])$.

4.1. Theorem. Let $\pi: A \rightarrow \mathbb{B}(\mathfrak{H})$ be an irreducible representation of a $C^{*}$-algebra A. If $q$ is a finite-dimensional projection on $\mathfrak{H}$ and $x$ is a self-adjoint contraction in $\mathbb{B}(q \mathfrak{H})=q \mathbb{B}(\mathfrak{H}) q$, then there is a self-adjoint contraction a in $A$ such that $\pi(a) q=x$ (and so also $x=q \pi(a))$ ).

In fact, if $x$ is positive or unitary (but being an exponential, necessarily), or a product of these (this covers all contractions in $\mathbb{B}(q \mathfrak{H})$ ), then $a$ can be chosen of the same type. Using the Glimm-Kadison result, we have the following Projective Transitivity Theorem which subsumes the earlier versions.

4.2. Theorem. Let $P$ be a projective $C^{*}$-algebra and $\pi: A \rightarrow \mathbb{B}(\mathfrak{H})$ an irreducible representation. If $q$ is a finite-dimensional projection on $\mathfrak{H}$ and $\theta: P \rightarrow \mathbb{B}(q \mathfrak{H})$ is a representation of $P$, there is a morphism $\varphi: P \rightarrow A$ such that

$$
\pi(\varphi(x)) q=\theta(x), \quad(x \in P) .
$$

(and so also $\theta(x)=q \pi(\varphi(x))$ ).

Proof. Let $\left\{x_{g} \in P \mid g \in G\right\}$ be a generating set of self-adjoint contractions for $P$. By the Glimm-Kadison transitivity theorem there is for each $g$ in $G$ a self-adjoint contraction $a_{g}$ in $A$ with $\pi\left(a_{g}\right) q=\theta\left(x_{g}\right)$. Consider now the universal $C^{*}$-algebra

$$
B=C^{*}\left\langle G \mid\|g\| \leq\left\|a_{g}\right\|, g \in G\right\rangle,
$$

cf. Section 2. By universality there exists three morphisms

$$
\begin{aligned}
& \alpha: B \rightarrow \mathbb{B}(q \mathfrak{H}) \oplus \mathbb{B}((1-q) \mathfrak{H}), \\
& \beta: B \rightarrow P \\
& \gamma: B \rightarrow A
\end{aligned}
$$


given by

$$
\begin{aligned}
& \alpha(g)=\pi\left(a_{g}\right), \\
& \beta(g)=x_{g}, \\
& \gamma(g)=a_{g} .
\end{aligned}
$$

With $\rho(x \oplus y)=x$ we then have a commuting diagram

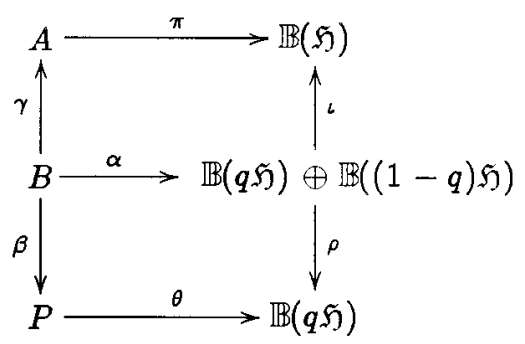

Since $P$ is projective, there is a lifting morphism $\sigma: P \rightarrow B$, with $\beta \circ \sigma=$ id. Define $\varphi=\gamma \circ \sigma$. Then for each $g$ in $G$

$$
\pi\left(\varphi\left(x_{g}\right)\right)=\pi \circ \gamma \circ \sigma\left(x_{g}\right)=\alpha\left(\sigma\left(x_{g}\right)\right),
$$

which must commute with $q$. Furthermore,

$$
\pi\left(\varphi\left(x_{g}\right)\right) q=\alpha\left(\sigma\left(x_{g}\right)\right) q=\rho \circ \alpha \circ \sigma\left(x_{g}\right)=\theta\left(x_{g}\right) .
$$

This completes the proof, since the $x_{g}$ 's generate $P$.

With $P=\mathbf{C} \mathbb{C}\left(=C_{0}(10,1]\right)$ we recover the version of Theorem 4.1 where $x$ is assumed to be positive and $a$ is required to be positive. With $P=\mathbb{C M}_{2}$ we recover a result of Glimm which states that if $\xi$ and $\eta$ are orthogonal unit vectors in $\mathfrak{H}$, and $\pi: A \rightarrow \mathbb{B}(\mathfrak{H})$ is irreducible, there is an $a$ in $A$ with $a^{2}=0,\|a\|=1$ and $\pi(a) \xi=\eta$. (Recall that $\mathbf{C M}_{2}$ is the universal $C^{*}$-algebra for the relation $x^{2}=0$; cf. Section 2.) Glimm used this result to produce a sufficient supply of nilpotents of order two inside an antiliminary $C^{*}$-algebra. So shall we.

The following notation will be used in the sequel: if $x$ and $y$ are positive elements in a $C^{*}$-algebra, we write $x \ll y$ if $x y=x$. Necessarily this means that $x$ and $y$ commute, and in the function algebra $C^{*}(x, y)$ every character $\gamma$ with $\gamma(x) \neq 0$ must have $\gamma(y)=1$. The concept $x \ll y$ is implicit in many of our previous proofs (notably in 3.9 and 3.10 ), and it is worth noting that the relation $x \ll y$ is one of the few liftable relations that preserve commutativity.

4.3. Lemma. If $A$ is an antiliminary $C^{*}$-algebra, there are elements $x, h$ in $A$ such that

$$
\begin{aligned}
\|x\| & =\|h\|=1, \\
x^{2} & =0, \\
h & \geq 0, \\
x^{*} x & \gg h .
\end{aligned}
$$

Proof. Being antiliminary, $A$ has an irreducible representation $\pi: A \rightarrow \mathbb{B}(\mathfrak{H})$ with $\operatorname{dim}(\mathfrak{H})=\infty$. Take a two-dimensional projection $q$ on $\mathfrak{H}$ and define $\theta: \mathbf{C M}_{2} \rightarrow$ 
$\mathbb{B}(q \mathfrak{H})$ by $\theta(f)=f(1)$ (identifying $\mathbb{M}_{2}$ with $\left.\mathbb{B}(q \mathfrak{H})\right)$ ). By the Projective Transitivity Theorem there is a morphism $\varphi: \mathbf{C M}_{2} \rightarrow A$ such that

$$
\pi\left(\varphi\left(g \otimes e_{i j}\right)\right) q=\theta\left(g \otimes e_{i j}\right)=g(1) \otimes e_{i j}
$$

for all $g$ in $C_{0}([0,1])$ and all matrix units $e_{i j}$. Let

$$
\begin{array}{lll}
g_{1}(t)=2 t, & g_{2}(t)=0 & \text { for } 0<t \leq \frac{1}{2} \\
g_{1}(t)=1, & g_{2}(t)=2 t-1 & \text { for } \frac{1}{2} \leq t \leq 1 .
\end{array}
$$

The two desired elements are then defined by

$$
x=\varphi\left(g_{1} \otimes e_{21}\right), \quad h=\varphi\left(g_{2} \otimes e_{11}\right) .
$$

Evidently the lemma above uses the antiliminarity of $A$ only in a rather superficial way, i.e. $A$ having at least one irreducible representation which is not a character. However, in applications the lemma will be applied to arbitrarily small hereditary $C^{*}$-subalgebras of $A$, and the full force of antiliminarity will be needed.

We shall later remark on the benefits of replacing $\mathbf{C M}_{2}$ by $\mathbf{C M}_{n}, n>2$. For now, we give a short proof of a result which is also, at least implicitly, due to Glimm.

4.4. Proposition. If $A$ is an antiliminary $C^{*}$-algebra, there exists a sequence $\left(x_{n}\right)$ in A such that, for all $n$,

$$
\begin{aligned}
\left\|x_{n}\right\| & =1, \\
x_{n}^{2} & =0, \\
x_{n}^{*} x_{n} & \gg x_{n+1}^{*} x_{n+1}, \\
x_{n}^{*} x_{n} & \gg x_{n+1} x_{n+1}^{*} .
\end{aligned}
$$

Proof. By Lemma 4.3 we have $x_{1}$ and $h_{1}$ in $A$ of norm one, with

$$
x_{1}^{2}=0, \quad h_{1} \geq 0, \quad x_{1}^{*} x_{1} \gg h_{1} .
$$

But $x_{1}^{*} x_{1} \gg h_{1}$ and $x_{2} \in \overline{h_{1} A h_{1}}$ imply that $x_{1}^{*} x_{1} \gg x_{2}^{*} x_{2}$ and $x_{1}^{*} x_{1} \gg x_{2} x_{2}^{*}$. An iterative process now produces the sequence $\left(x_{n}\right)$ (together with the auxiliary sequence $\left.\left(h_{n}\right)\right)$.

We now only have to discover what kind of $C^{*}$-algebra is generated by a sequence of nilpotents as above, and we will have recovered Glimm's celebrated result that the Fermion algebra is a quotient of some $C^{*}$-subalgebra of $A$, with the advantage that we will now know something about the form of the subalgebra.

4.5. Proposition. The universal $C^{*}$-algebra $B_{n}$ generated by elements $x_{1}, \ldots, x_{n}$, subject to the relations

$$
\begin{array}{rlrl}
\left\|x_{j}\right\| & \leq 1, & & (1 \leq j \leq n), \\
x_{j}^{2} & =0 & & (1 \leq j \leq n), \\
x_{j}^{*} x_{j} & \gg x_{k}^{*} x_{k}, & \\
x_{j}^{*} x_{j} \gg x_{k} x_{k}^{*}, & & (j<k),
\end{array}
$$

is isomorphic to the mapping telescope $\mathbf{T}\left(\mathbb{M}_{2}, \mathbb{M}_{4}, \ldots, \mathbb{M}_{2^{n}}\right)$. Regarding the telescope as a subalgebra of $\left.\left.C_{0}(] 0, n\right]\right) \otimes \mathbb{M}_{2} \otimes \mathbb{M}_{2} \otimes \cdots \otimes \mathbb{M}_{2}$, the isomorphism is given 
by

$$
x_{j} \mapsto f_{j} \otimes \underbrace{e_{11} \otimes \cdots \otimes e_{11}}_{j-1} \otimes e_{21} \otimes \underbrace{1 \otimes \cdots \otimes 1}_{n-j},
$$

where

$$
\begin{aligned}
f_{j}(t) & =((t+1-j) \vee 0) \wedge 1 \\
& = \begin{cases}0, & 0<t \leq j-1, \\
t+1-j, & j-1 \leq t \leq j, \\
1, & j \leq t \leq n .\end{cases}
\end{aligned}
$$

Proof. That we obtain a surjective morphism from $B_{n}$ onto the finite telescope follows from universality; cf. Section 2 . To prove injectivity it suffices to show that every irreducible representation of $B_{n}$ is the pull-back of an irreducible representation of $\mathbf{T}\left(\mathbb{M}_{2}, \ldots, \mathbb{M}_{2^{n}}\right)$. Equivalently, we show by induction that the only irreducible representations of the relations $(*)$ are those of the form

$$
x_{j}=\alpha_{j} \underbrace{e_{11} \otimes \cdots \otimes e_{11}}_{j-1} \otimes e_{21} \otimes \underbrace{1 \otimes \cdots \otimes 1}_{n-j}
$$

in $\mathbb{B}\left(\mathbb{C}^{2} \otimes \mathbb{C}^{2} \otimes \cdots \otimes \mathbb{C}^{2}\right)$, where the scalars $\alpha_{j}$ satisfy

$$
\begin{aligned}
\alpha_{1} & =\alpha_{2}=\cdots=\alpha_{n-1}=1, \\
0 & <\alpha_{k}<1, \\
\alpha_{n+1} & =\cdots=\alpha_{n}=0,
\end{aligned}
$$

for some $k$.

The case $n=1$ is just a restatement of the fact that $\mathrm{CM}_{2}$ is universal for the relations $x^{2}=0,\|x\| \leq 1$. Now suppose that we have proved the assertion for all $(n-1)$-tuples, and take $x_{1}, \ldots, x_{n}$ in $\mathbb{B}(\mathfrak{H})$ satisfying $(*)$, such that

$$
C^{*}\left(x_{1}, \ldots, x_{n}\right)^{\prime}=\mathbb{C} 1 .
$$

The operator $x_{1}^{*} x_{1}+x_{1} x_{1}^{*}$ commutes with all the $x_{j}$ 's, hence with $C^{*}\left(x_{1}, \ldots, x_{n}\right)$, and so $x_{1}^{*} x_{1}+x_{1} x_{1}^{*}=\alpha 1$, where $0 \leq \alpha \leq 1$. If $\alpha<1$, the relations

$$
1>x_{1}^{*} x_{1} \gg x_{j}^{*} x_{j} \text { for } 2 \leq j \leq n
$$

force $x_{2}=x_{3}=\cdots=x_{n}=0$. This reduces to the known case, $n=1$. If $\alpha=1$, we let

$$
e_{11}=x_{1}^{*} x_{1}, \quad e_{12}=x_{1}^{*}, \quad e_{21}=x_{1}, \quad e_{22}=x_{1} x_{1}^{*} .
$$

These elements act like matrix units, and $x_{j}=e_{11} x_{j} e_{11}$ for all $j>1$. Working in matrix notation, we write

$$
\begin{aligned}
& x_{1}=\left(\begin{array}{ll}
0 & 0 \\
1 & 0
\end{array}\right), \\
& x_{j}=\left(\begin{array}{cc}
y_{j} & 0 \\
0 & 0
\end{array}\right), \quad(2 \leq j \leq n) .
\end{aligned}
$$

Then the elements $y_{2}, \ldots, y_{n}$ are seen to be an irreducible representation of the relations $(*)$ on $e_{11} \mathfrak{H}$ for $n-1$. By induction the $y_{j}$ 's, and thus also $x_{1}, \ldots, x_{n}$, are of the desired form.

We denote by $\mathbf{T}\left(\mathbb{M}_{2 \infty}\right)$ the mapping telescope corresponding to the inductive system $\mathbb{M}_{2} \subset \mathbb{M}_{4} \subset \cdots \subset \mathbb{M}_{2^{n}} \subset \cdots \subset \mathbb{M}_{2^{\infty}}$. 
4.6. Theorem. The telescope $\mathbf{T}\left(\mathbb{M}_{2 \infty}\right)$ is the universal $C^{*}$-algebra generated by elements $x_{1}, x_{2}, \ldots$, subject to the relations

$$
\begin{aligned}
\left\|x_{n}\right\| & \leq 1, \\
x_{n}^{2} & =0, \\
x_{n}^{*} x_{n} & \gg x_{n+1}^{*} x_{n+1}, \\
x_{n}^{*} x_{n} & \gg x_{n+1} x_{n+1}^{*} .
\end{aligned}
$$

Proof. Clearly the universal $C^{*}$-algebra is the inductive limit of the algebras $B_{n}$ from the previous proposition, whereas the telescope $\mathbf{T}\left(\mathbb{M}_{2} \infty\right)$ is the inductive limit of the finite telescopes (cf. Section 2). The result is therefore immediate from Proposition 4.5.

Since the Fermion algebra $\mathbb{M}_{2 \infty}$ is simple, the primitive spectrum of $\mathbf{T}\left(\mathbb{M}_{2 \infty}\right)$ is homeomorphic to $] 0, \infty$ ] (where the point $\infty$ supports all the irreducible representations of $\mathbb{M}_{2 \infty}$ !). In particular, the quotients of $\mathbf{T}\left(\mathbb{M}_{2 \infty}\right)$ are found by restriction to a closed subset $Z$ of $] 0, \infty]$. Only if $\infty \in Z$ will all the generators have norm one. So Proposition 4.4 in conjunction with Theorem 4.6 yield our version of Glimm's theorem:

4.7. Theorem. If $A$ is an antiliminary $C^{*}$-algebra, it contains a $C^{*}$-subalgebra isomorphic to

$$
\left.\left.\left\{f \in C_{0}\left(Z, \mathbb{M}_{2^{\infty}}\right) \mid f(t) \in \mathbb{M}_{2^{n}} \text { if } t \in Z \cap\right] 0, n\right]\right\}
$$

for some closed subset $Z$ of $] 0, \infty]$ containing $\infty$.

4.8. Remark. In [20] (cf. [21, 6.7.3]), Glimm's theorem was extended from the Fermion algebra to arbitrary $U H F$-algebras. We wish to point out that our method also covers this generalization. Choose a sequence $m(1), m(2), \ldots$ of natural numbers greater than one, and put $m(n) !=\prod_{k=1}^{n} m(k)$. Then consider the inductive system

$$
\mathbb{M}_{m(1) !} \subset \mathbb{M}_{m(2) !} \subset \ldots,
$$

where the embeddings are given by writing

$$
\mathbb{M}_{m(n) !}=\mathbb{M}_{m(n-1) !} \otimes \mathbb{M}_{m(n)} .
$$

The inductive limit is the $U H F$-algebra (or Glimm algebra) $\mathbb{M}_{m(\infty) !}$. Corresponding to each $U H F$-algebra we have a mapping telescope $\mathbf{T}\left(\mathbb{M}_{m(\infty) !}\right)$.

It is straightforward to show - mimicking the proof of Proposition 4.5 - that $\mathbf{T}\left(\mathbb{M}_{m(\infty) !}\right)$ is the universal $C^{*}$-algebra for a sequence of generators $x_{j, m(n)}, 2 \leq$ $j \leq m(n), n \in \mathbb{N}$ (a sequence of finite sets, really), subject to the relations

$$
\begin{aligned}
\left\|x_{j, m(n)}\right\| & \leq 1, & & \\
x_{j, m(n)} x_{k, m(n)} & =0, & & 2 \leq j, k \leq m(n), \\
x_{j, m(n)}{ }^{*} x_{j, m(n)} & =x_{k, m(n)}{ }^{*} x_{k, m(n)}, & & 2 \leq j, k \leq m(n), \\
x_{j, m(n)}^{*} x_{j, m(n)} & \gg x_{k, m(n+1)}^{*} x_{k, m(n+1)}, & & \text { for all } j, k, n, \\
x_{j, m(n)}^{*} x_{j, m(n)} & \gg x_{k, m(n+1)} x_{k, m(n+1)}^{*}, & & \text { for all } j, k, n \\
x_{j, m(n)}^{*} x_{k, m(n)} & =0, & & \text { if } j \neq k .
\end{aligned}
$$


The notation and the implications can be found in $[21,6.6]$, but it suffices to note that for each $n$, the elements

$$
x_{2, m(n)}, x_{3, m(n)}, \ldots, x_{m(n), m(n)}
$$

are (multiples of) the first column in $\mathbb{M}_{m(n)}$, except for the deleted (1,1)-element.

To extend Theorem 4.7 from $\mathbb{M}_{2 \infty}$ to $\mathbb{M}_{m(n)}$ ! we just have to extend Lemma 4.3 (Proposition 4.4 will apply, mutatis mutandis) and show that every antiliminary $C^{*}$-algebra $A$ contains elements $x_{2}, x_{3}, \ldots, x_{m}$ and $h$ such that

$$
\begin{array}{ll}
\left\|x_{j}\right\|=\|h\|=1, & 2 \leq j \leq m, \\
x_{j} x_{k}=0, & \text { all } j, k, \\
x_{j}^{*} x_{j}=x_{k}^{*} x_{k}, & \text { all } j, k, \\
x_{j}^{*} x_{j} \gg h, & \text { all } j, \\
x_{j} x_{k}=0, & \text { if } j \neq k .
\end{array}
$$

This is done by replacing $\mathbf{C M}_{2}$ by $\mathbf{C M}_{m}$ in the proof of Lemma 4.3.

4.9. Remark. We will show later, in Theorem 7.2, that every AF-telescope is projective. This means that in Theorem 4.7 (and its generalization hinted at in Remark 4.8) we may replace the condition that $A$ is antiliminary, by the weaker condition that $A$ is not of type I. For in the latter case $A$ has a non-zero antiliminary quotient.

The presentation of $\mathbf{T}\left(\mathbb{M}_{2 \infty}\right)$ given in Theorem 4.6 is not related to the Fermion picture of $\mathbb{M}_{2 \infty}$. There is such a presentation, and though we have no immediate use for it, we display it for its elegance. For completeness we first state the following well-known fact.

4.10. Lemma. The universal $C^{*}$-algebra with generators $\left\{a_{j} \mid j \leq n\right\}$, where $1 \leq n \leq \infty$, subject to the relations

$$
\begin{array}{ll}
a_{j} a_{k}+a_{k} a_{j}=0, & \text { all } j, k, \\
a_{j}^{*} a_{k}+a_{k} a_{j}^{*}=\delta_{j k} 1, & \text { all } j, k,
\end{array}
$$

is isomorphic to $\mathbb{M}_{2^{n}}=\mathbb{M}_{2} \otimes \cdots \otimes \mathbb{M}_{2}$ via the map

$$
a_{j} \mapsto \underbrace{v \otimes \cdots \otimes v}_{j-1} \otimes e_{21} \otimes \underbrace{1 \otimes \cdots \otimes 1}_{n-j}
$$

where $v=e_{22}-e_{11}$.

Proof. For $n<\infty$ this is pure (linear) algebra. For $n=\infty$ it follows from the description of the Fermion algebra $\mathbb{M}_{2 \infty}$ as the inductive limit of the matrix algebras $\mathbb{M}_{2^{n}}$.

4.11. Theorem. The Fermion telescope $\mathbf{T}\left(\mathbb{M}_{2 \infty}\right)$ is the universal $C^{*}$-algebra generated by a sequence $\left(a_{n}\right)$ of contractions, subject to the relations

$$
\begin{array}{rlrl}
a_{m} a_{n}+a_{n} a_{m} & =0, & & \text { all } n, m, \\
a_{m}^{*} a_{n}+a_{n} a_{m}^{*}=0, & & \text { if } n \neq m, \\
a_{m}^{*} a_{m}+a_{m} a_{m}^{*} \gg a_{n}^{*} a_{n}+a_{n} a_{n}^{*}, & & \text { if } m<n .
\end{array}
$$

Proof. Let $B$ denote the universal $C^{*}$-algebra generated by the sequence $\left(a_{n}\right)$. Note now that each element

$$
h_{n}=a_{n}^{*} a_{n}+a_{n} a_{n}^{*}
$$


commutes with all $a_{m}$, and thus is central in $B$. Moreover,

$$
0 \leq h_{n} \leq 1
$$

and

$$
h_{m} \gg h_{n} \quad \text { if } m<n .
$$

Therefore, if $\pi: B \rightarrow \mathbb{B}(\mathfrak{H})$ is an irreducible representation of $B$, and if we put $b_{n}=\pi\left(a_{n}\right)$ and $k_{n}=\pi\left(h_{n}\right)$, there are two cases: either for some $n$ and some $\alpha$ in ] $0,1[$ we have

$$
\begin{aligned}
k_{m} & =1 \quad \text { for } m<n, \\
k_{n} & =\alpha 1, \\
k_{m} & =0 \quad \text { for } m>n,
\end{aligned}
$$

or else we have $k_{n}=1$ for all $n$.

In the first case the relations force $b_{j}=0$ for $j>n$, and the elements

$$
b_{1}, b_{2}, \ldots, b_{n-1}, \alpha^{-1 / 2} b_{n}
$$

now satisfy the standard Fermion relations, which by Lemma 4.10 means that $\pi(B)=\mathbb{M}_{2^{n}}$. If $\varphi: B \rightarrow \mathbf{T}\left(\mathbb{M}_{2^{\infty}}\right)$ is the surjective morphism determined by

$$
\varphi\left(a_{j}\right)=f_{j} \otimes \underbrace{v \otimes \cdots \otimes v}_{j-1} \otimes e_{21} \otimes 1 \otimes \ldots,
$$

where $f_{j}(t)=((t+1-j) \vee 0) \wedge 1$, then we see from Lemma 4.10 that $\pi$ is the pull-back of $\varphi$ by evaluation at $t=n-1+\alpha^{1 / 2}$.

In the second case the sequence $\left(b_{n}\right)$ satisfies the Fermion relations, and, again from Lemma 4.10 we see that $\pi$ is the pull-back of a subrepresentation of $\varphi$ by evaluation at $t=\infty$.

We have shown that every irreducible representation of $B$ is a pull-back of $\varphi$, which proves that $\varphi$ is an isomorphism.

\section{Multiplier Realization Theorems}

Recall from $[21,3.12]$ that every non-unital $C^{*}$-algebra $E$ is embedded as an essential ideal in its multiplier algebra $M(E)$, and $M(E)$ is the universal $C^{*}$-algebra with this property, being the non-commutative analogue of the Stone-Čech compactification. The corona algebra $C(E)=M(E) / E$ has many exciting properties (the $S A W^{*}$-property, the asymptotically abelian, countable Riesz separation property, etc.; see [19] or [21]) which facilitate liftings from $C(E)$ to $M(E)$. The fact - already mentioned - that a $C^{*}$-algebra $P$ is projective if it is merely corona projective makes these properties important for our study.

5.1. Theorem. Let $A$ and $E$ be $\sigma$-unital $C^{*}$-algebras and $\varphi: A \rightarrow C(E)$ a morphism of $A$ into the corona algebra of $E$. If $P$ is a projective $C^{*}$-algebra, then for every morphism $\theta: P \rightarrow M(A)$ there is a morphism $\psi: P \rightarrow M(E)$ such that for all $p$ in $P$ and $a$ in $A$

$$
\pi(\psi(p)) \varphi(a)=\varphi(\theta(p) a),
$$

where $\pi: M(E) \rightarrow C(E)$ is the quotient map. 
Proof. Consider the following diagram:

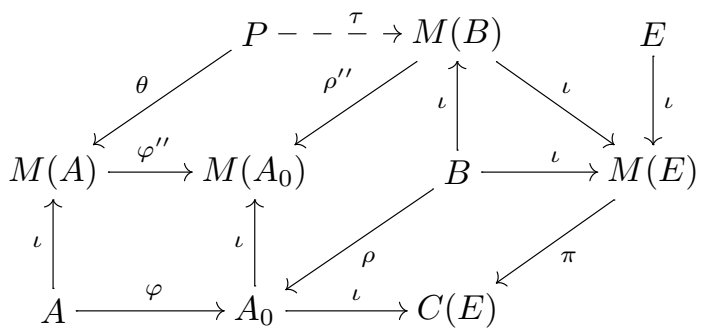

Here $A_{0}=\varphi(A)$, so that $\iota: A_{0} \rightarrow C(E)$ is an inclusion. Moreover, since $A$ is $\sigma$-unital, there is, by [22] and [10], a canonical surjective morphism $\varphi^{\prime \prime}: M(A) \rightarrow$ $M\left(A_{0}\right)$, extending $\varphi$. Let $B=\pi^{-1}\left(A_{0}\right)$, so that we have a surjective morphism $\rho: B \rightarrow A_{0}$, making the part of the diagram involving $A_{0}, B, M(E)$ and $C(E)$ commutative. Since $E \subset B \subset M(E)$, so that $E$ is an essential ideal in $B$, we have an embedding $\iota: M(B) \rightarrow M(E)$.

Note that since both $A$ and $E$ are $\sigma$-unital, so is $B$. In fact, any element $h+k$ will be strictly positive for $B$ if $h$ is strictly positive for $E$ and $\rho(k)$ is strictly positive for $A_{0}$. Since $\rho$ is a surjective morphism there is therefore a canonical surjective morphism $\rho^{\prime \prime}: M(B) \rightarrow M\left(A_{0}\right)$ extending $\rho$. Consider now the morphism $\varphi^{\prime \prime} \circ \theta: P \rightarrow M\left(A_{0}\right)$. Since $P$ is projective, this lifts to a morphism $\tau: P \rightarrow M(B)$, such that $\rho^{\prime \prime} \circ \tau=\varphi^{\prime \prime} \circ \theta$. Define $\psi=\iota \circ \tau$ as a morphism from $P$ into $M(E)$. If $p \in P$ and $a \in A$, then since $\varphi(a)=\rho(b)$ for some $b$ in $B$ we get, by diagram chasing,

$$
\begin{aligned}
\pi(\psi(p)) \varphi(a) & =(\pi \circ \iota \circ \tau(p)) \rho(b)=\pi(\iota \circ \tau(p) \iota(b)) \\
& =\pi(\tau(p) b)=\rho(\tau(p) b)=\rho^{\prime \prime}(\tau(p)) \rho(b) \\
& =\varphi^{\prime \prime}(\theta(p)) \varphi(a)=\varphi(\theta(p) a),
\end{aligned}
$$

as desired.

5.2. Corollary. If $A$ is the split extension of $P$ by $Q$, where $P$ is $\sigma$-unital, and if $Q$ is projective, then every morphism $\varphi$ of $P$ into a corona algebra $C(E)$ of a $\sigma$-unital $C^{*}$-algebra $E$ extends to a morphism $\widetilde{\varphi}: A \rightarrow C(E)$.

Proof. Let $\theta: Q \rightarrow M(P)$ be the morphism that determines $A$ (cf. Proposition 2.1), and apply Theorem 5.1 to obtain a morphism $\varphi_{0}: Q \rightarrow C(E)$ such that $\varphi_{0}(q) \varphi(p)=\varphi(\theta(q) p)$ for all $q$ in $Q$ and $p$ in $P$. By Proposition 2.2 the pair $\left(\varphi, \varphi_{0}\right)$ determines a morphism $\widetilde{\varphi}: A \rightarrow C(E)$.

5.3. Theorem. If $A$ is a projective $C^{*}$-algebra which is written as a (split) extension $P \rightarrow A \rightarrow Q$, where also $Q$ is projective, then $P$ is projective.

Proof. Let $\varphi: P \rightarrow C(E)$ be a morphism of $P$ into the corona algebra of some $\sigma$-unital $C^{*}$-algebra $E$. By Corollary 5.2 we have an extension $\widetilde{\varphi}: A \rightarrow C(E)$, and since $A$ is projective this lifts to a morphism $\widetilde{\psi}: A \rightarrow M(E)$ such that $\pi \circ \widetilde{\psi}=\widetilde{\varphi}$. Now let $\psi=\left.\widetilde{\psi}\right|_{P}$ to obtain the desired lifting of $\varphi$.

For later use (in section 7) we give an application of the previous theorem.

5.4. Proposition. Let $A=\overline{\bigcup A_{n}}$ be an inductive limit of $C^{*}$-algebras, and, with $\sim$ denoting (forced) unitization, consider $\widetilde{A}$ as the inductive limit $\overline{\bigcup \widetilde{A}_{n}}$. If $\mathbf{T}(\widetilde{A})$ is projective then so is $\mathbf{T}(A)$. 
Proof. We leave it to the reader to establish that there is an exact sequence

$$
0 \rightarrow \mathbf{T}(A) \rightarrow \mathbf{T}(\widetilde{A}) \rightarrow \mathbf{T}(\mathbb{C}) \rightarrow 0 .
$$

Here $T(\mathbf{C})$ means the telescope for the system

$$
\mathbb{C} \stackrel{\text { id }}{\longrightarrow} \mathbb{C} \stackrel{\text { id }}{\longrightarrow} \ldots
$$

However, $\mathbf{T}(\mathbb{C}) \cong \mathbf{C} \mathbb{C} ;$ so, again, Theorem 5.3 applies.

At this stage the reader must have asked - and possibly solved - the question whether (split) extensions of projective $C^{*}$-algebras are again projective. Certainly our telescopic examples support this hypothesis. Unfortunately it is not true.

5.5. Example. Let $X$ be the closure of the set

$$
G=\left\{\left(t, \sin t^{-1}\right) \mid 0<t \leq 1\right\}
$$

in $\mathbb{R}^{2}$ with the point $(0,1)$ removed. The intersection of $X$ with the $Y$-axis is a closed set $F$, homeomorphic with $] 0,1]$. The rest is $X \backslash F=G$, which is also homeomorphic to $] 0,1]$ (projecting on the $X$-axis). We have therefore a short exact sequence

$$
C_{0}(G) \rightarrow C_{0}(X) \rightarrow C_{0}(F)
$$

where both the ideal and the quotient are projective, being isomorphic to $\mathbf{C} \mathbb{C}$. But $C_{0}(X)$ is not projective, because $X$ is not an absolute retract. If it were, there would be a continuous map $f: \widehat{X} \rightarrow X$, where $\widehat{X}$ denotes the cone of $X$, such that $f(x, 1)=x$ for each $x$ in $X$. (This corresponds to making $C_{0}(X)$ a subalgebra of $\mathbf{C} C_{0}(X)$, lifting the morphism $g \rightarrow g(1)$ of $\mathbf{C} C_{0}(X)$ onto $C_{0}(X)$.) The definition $f_{t}(x)=f(x, t)$ shows that $X$ is contractible, and in particular arcwise connected. But $X$ is the standard example of a (connected) not arcwise connected set.

In the positive direction one can show, rather easily, that an extension of projective $C^{*}$-algebras is residually finite-dimensional. A $C^{*}$-algebra is residually finitedimensional if it embeds into $\prod M_{n_{k}}$ for some sequence of natural numbers $n_{k}$. If a $C^{*}$-algebra embeds into $\prod M_{n_{k}} / \bigoplus M_{n_{k}}$, then it is an $M F$ algebra. This is not the definition of an $M F$, or matricial field, algebra, but it is equivalent by [3, Theorem $3.2 .2]$.

5.6. Theorem. If $I$ is an $M F$ algebra and $P$ is projective, and $A$ is an extension of $I$ by $P$, then $A$ is an $M F$ algebra.

Proof. By the assumption on $I$, there exists an injective morphism $\varphi_{0}: I \rightarrow C(E)$ where $E=\bigoplus M_{n_{k}}$. Corollary 5.2 implies that there is an extension of $\varphi_{0}$ to a morphism $\varphi: A \rightarrow C(E)$.

As noted in the introduction, the projectivity of $P$ implies that it is residually finite-dimensional. Taking an infinite family of finite-dimensional representations, each occurring infinitely often, we obtain an embedding $\psi_{0}: P \rightarrow C(F)$, where $F=\bigoplus M_{r_{k}}$. Using a splitting $\lambda: P \rightarrow A$ we obtain a morphism $\psi: A \rightarrow C(F)$, so that now $\varphi \oplus \psi: A \rightarrow C(E) \oplus C(F)$ is injective. Since

$$
C(E) \oplus C(F) \subseteq C\left(\bigoplus M_{n_{k}+r_{k}}\right),
$$

we have proven that $A$ is also $M F$. 


\section{Presentations of Telescopes}

As a prerequisite for showing that every $A F$-telescope is a projective $C^{*}$-algebra we need a detailed study of its presentations. A glance at the problem will explain why. To lift a morphism from an infinite telescope, one must work inductively and lift the cone $\mathbf{C} A_{n}$, having already lifted the finite telescope $\mathbf{T}\left(A_{1}, \ldots, A_{n-1}\right)$ (cf. Proposition 2.4). This means lifting an ideal, given the constraints of having lifted the quotient! Only the most careful labeling of the embeddings $A_{n} \hookrightarrow A_{n+1}$ will make this process possible. The model we present uses graph-theoretic language instead of numbers, and the groupoid of paths in the diagram will be our chosen object.

Let $S$ be a finite set of cardinality \#S, equipped with an equivalence relation $\sim$. Choose a set $[S]$ of representatives for the equivalence classes, and let $e \mapsto[e]$ denote the selection function (so that $e \sim[e] \in[S]$ ). Finally, let $\#[e]$ denote the cardinality of the equivalence class represented by $[e]$.

6.1. Lemma. With notations as above, the mapping cone for the $C^{*}$-algebra $\bigoplus \mathbb{M}_{\#[e]}$, i.e. the algebra $\bigoplus \mathbf{C M}_{\#[e]}$, is the universal $C^{*}$-algebra with generators $[S]$ $[S]$ $\left\{x_{e} \mid e \in S\right\}$, subject to the relations

$$
\begin{aligned}
\left\|x_{e}\right\| & \leq 1 & & \text { for all } e \text { in } S, \\
x_{e} x_{f} & =0 & & \text { if }[e] \neq f, \\
x_{e}^{*} x_{f} & =0 & & \text { if } e \neq f, \\
x_{e} x_{f}^{*} & =0 & & \text { if }[e] \neq[f], \\
x_{e}^{*} x_{e} & =x_{f}^{*} x_{f} & & \text { if }[e]=[f], \\
x_{[e]} & =\left(x_{[e]}^{*} x_{[e]}\right)^{1 / 2} & & \text { for all }[e] \text { in }[S] .
\end{aligned}
$$

Proof. For a single equivalence class this is just a reformulation of $[16,2.7]$. The general case follows from the usual properties of direct sums.

To describe in detail the isomorphism of the universal $C^{*}$-algebra generated by the $x_{e}$ 's with the mapping cone, note first that $\bigoplus \mathbb{M}_{\#[e]}$ is linearly spanned by generalized matrix units

$$
\left\{v_{e, f} \mid(e, f) \in S^{2} \text { and }[e]=[f]\right\}
$$

satisfying the rules

$$
\begin{aligned}
v_{e, f}^{*} & =v_{f, e}, \\
v_{e, f} v_{g, h} & =\delta_{f, g} v_{e, h} .
\end{aligned}
$$

Identifying $\mathbf{C}\left(\bigoplus \mathbb{M}_{\#[e]}\right)$ with $\left.\left.C_{0}(] 0,1\right]\right) \otimes\left(\bigoplus \mathbb{M}_{\#[e]}\right)$ as usual, the isomorphism of the universal $C^{*}$-algebra to the mapping cone is given by

$$
x_{e} \mapsto \mathrm{id} \otimes v_{e,[e]}
$$

The inverse map is defined on a set of elements with dense span by

$$
\left.\left.\gamma\left(t^{2}\right) t^{2} v_{e, f} \mapsto x_{e} \gamma\left(x_{e}^{*} x_{e}\right) x_{f}^{*} \quad\left(\gamma \in C_{0}(] 0,1\right]\right)\right)
$$

6.2. Remark. With just one set of extra relations:

$$
x_{[e]}^{2}=x_{[e]} \quad([e] \in[S]),
$$


the lemma above becomes a presentation of $\bigoplus \mathbb{M}_{\#[e]}$, because now all the $x_{e}$ become partial isometries. Note also that we have deliberately introduced the "unnecessary" generators $x_{[e]}$. This small redundance in our model is amply compensated for by its elegance.

We now, for the rest of the section, fix a Bratteli diagram (with multiple embeddings represented by multiple edges) corresponding to a unital AF system

$$
\mathbb{C} 1 \subseteq A_{1} \subseteq A_{2} \subseteq A_{3} \subseteq \cdots .
$$

We shall obtain a presentation for

$$
\mathbf{T}(A)=\mathbf{T}\left(A_{1}, A_{2}, A_{3}, \ldots\right) .
$$

The algebra $\mathbb{C} 1$ is included in the $\mathrm{AF}$ system so that in the Bratteli diagram we have a single vertex at the zeroth level, which we call a root vertex. A key observation is that the weight of a vertex (= the size of the corresponding matrix algebra) equals the number of paths from the root to that vertex.

By the $n$th level of vertices we mean those connected to the root by a length- $n$ path, so these are the vertices that represent the factors of $A_{n}$. The total edge set we denote $E=\bigcup E_{n}$, and $e \in E_{n}$ means that $e$ is an edge that connect a level$(n-1)$ vertex $s(e)$ to a level- $n$ vertex $r(e)$. We call $s(e)$ the source of $e$ and $r(e)$ the range of $e$. (Ranges below; sources above.)

We shall write $\bar{e}=e_{1} e_{2} \cdots e_{n}$ for a downward (= away from the root, sadly) path along adjacent edges. We let $r(\bar{e})=r\left(e_{n}\right)$ and $s(\bar{e})=s\left(e_{1}\right)$, and shall define the composition of two paths $\bar{e}$ and $\bar{f}$ (denoted $\bar{e} \bar{f}$ ) if $r(\bar{e})=s(\bar{f})$.

With the above notation, the algebra $A_{n}$ is linearly spanned by the generalized matrix units $v_{\bar{e}, \bar{f}}$, where $\bar{e}$ and $\bar{f}$ range over all paths from the root to the $n$th level satisfying $r(\bar{e})=r(\bar{f})$. The rules for adjoints and multiplication are simple:

$$
v_{\bar{e}, \bar{f}^{*}}=v_{\bar{f}, \bar{e}}
$$

and

$$
v_{\bar{e}, \bar{f}} v_{\bar{g}, \bar{h}}=\delta_{\bar{f}, \bar{g}} v_{\bar{e}, \bar{h}} .
$$

The embedding $A_{n} \rightarrow A_{n+1}$ is determined by

$$
v_{\bar{e}, \bar{f}} \mapsto \sum v_{\bar{e} e_{n}, \bar{f} e_{n}},
$$

where the summation is over all edges $e_{n}$ in $E_{n+1}$ with $s\left(e_{n}\right)=r(\bar{e})(=r(\bar{f}))$.

If we identify each $v_{\bar{e}, \bar{f}}$ with its image in the $A F$-algebra $A=\overline{\bigcup A_{n}}$, we can define multiplication between matrix units in different subalgebras. A moment's reflection shows that the rules are

$$
v_{\bar{e}, \bar{f}} v_{\bar{g}, \bar{h}}=0
$$

unless $\bar{g}=\overline{f d}$ or $\bar{f}=\bar{g} \bar{d}$ for some path $\bar{d}$, in which case

$$
\begin{aligned}
v_{\bar{e}, \bar{f}} v_{\bar{f} \bar{d}, \bar{h}}=v_{\bar{e} \bar{d}, \bar{h}}, \\
v_{\bar{e}, \bar{g} \bar{d}} v_{\bar{g}, \bar{h}}=v_{\bar{e}, \bar{h} \bar{d}}
\end{aligned}
$$

(of course, $\bar{d}$ might be the zero path).

Because of these multiplication rules, if we have a subset of the $v_{\bar{e}, \bar{f}}$ 's that generates $A_{n-1}$, we need only add enough extra elements to account for the edges in $E_{n}$, in order to create a generating set for $A_{n}$. In fact, a minimal generating set for $A$ can be associated with the edges of the Bratteli diagram. (Well, almost minimal.) 
The construction is non-canonical, though, so we now mark some edges as being the "preferred" way back to the root.

More precisely, choose a subset $\left[E_{n}\right]$ of $E_{n}$ that contains exactly one edge adjacent to each vertex "below". Thus from each level- $n$ vertex there is exactly one way back through $\left[E_{n}\right]$ to some level- $n-1$ vertex. Let $e \mapsto[e]$ denote the (selection) function which sends an edge $e$ to the preferred edge in $[E]=\bigcup\left[E_{n}\right]$ that leads down to the same vertex $(r(e)=r([e]))$.

With the choice of $[E]$, each vertex determines a unique path through elements from $[E]$ from the root to that vertex. Therefore, each edge $e$ in $E_{n}$ is associated with two canonical paths $p(e)$ and $q(e)$, where $p(e)$ is the path from the root to $r(e)$ through $[E]$ and $q(e)$ is the path from the root to $r(e)$ of the form $\bar{e} e$, where $\bar{e}$ is a path through $[E]$.

6.3. Lemma. With notations as above, the $C^{*}$-algebra $A_{n}$ is generated by the set

$$
\left\{v_{q(e), p(e)} \mid e \in E_{1} \cup E_{2} \cup \cdots \cup E_{n}\right\} .
$$

Proof. For $n=1$ we have for each $e$ in $E_{1}$ that $p(e)=[e]$ and $q(e)=e$. That the set $v_{e,[e]}, e \in E_{1}$, generates $A_{1}$ follows from Remark 6.2. Assume now that we have established the result for some $A_{n}(n \geq 1)$, and consider $v_{\bar{e}, \bar{f}}$ in $A_{n+1}$. We can write $\bar{e}=\widehat{e} e$ and $\bar{f}=\widehat{f} f$ for some $e, f$ in $E_{n+1}$ with $r(e)=r(f)$. The multiplication rules for matrix units given above show that for any paths $\bar{h}$ and $\bar{k}$ from the root down to the $n$th level with $r(\bar{h})=s(e), r(\bar{k})=s(f)$ we have

$$
v_{\bar{e}, \bar{f}}=v_{\hat{e} e, \bar{f}}=v_{\hat{e}, \bar{h}} v_{\bar{h} e, \bar{f}}=v_{\hat{e}, \bar{h}} v_{\bar{h} e, \hat{f} f}=v_{\hat{e}, \bar{h}} v_{\bar{h} e, \bar{k} f} v_{\bar{k}, \hat{f}} .
$$

Here $v_{\hat{e}, \bar{h}}$ and $v_{\bar{k}, \hat{f}}$ belong to $A_{n}$ by assumption, and we choose $\bar{h}$ and $\bar{k}$ such that $\bar{h} e=q(e)$ and $\bar{k} f=q(f)$. Noting that $p(e)=p(f)$, because $r(e)=r(f)$, we see that

$$
v_{\bar{h} e, \bar{k} f}=v_{q(e), p(e)} v_{p(e), q(f)}=v_{q(e), p(e)} v_{q(f), p(f)}{ }^{*} .
$$

Combining these equations we see that $v_{\bar{e}, \bar{f}}$ is generated by matrix units of the form $v_{q(e), p(e)}, e \in E_{n+1}$, together with elements from $A_{n}$, and the proof is completed by induction.

With Lemma 6.3 in mind we construct generators of $\mathbf{T}(A)$ as follows: define a sequence $\left(\alpha_{n}\right)$ of functions in $\left.\left.C_{0}(] 0, \infty\right]\right)$ by

$$
\alpha_{n}(t)=((t+1-n) \vee 0) \wedge 1,
$$

so that each $\alpha_{n}$ is "the identity map" on the relevant interval ] $\left.n-1, n\right]$, and $\alpha_{n} \alpha_{m}=$ $\alpha_{m}$ if $n<m$. Then consider the elements

$$
\alpha_{n} \otimes v_{q(e), p(e)} \quad\left(e \in E_{n}, n \in \mathbb{N}\right) .
$$

With this model in mind we present the following.

6.4. Theorem. The mapping telescope $\mathbf{T}(A)$ for the $A F$-algebra $A$ is the universal $C^{*}$-algebra with generators $x_{e}, e \in E$, where $E=\bigcup E_{n}$ denotes the set of edges in the Bratteli diagram, equipped with an equivalence relation determined by a set 
$[E]=\bigcup\left[E_{n}\right]$ of preferred edges, subject to the relations

$$
\begin{aligned}
\left\|x_{e}\right\| & \leq 1 & & \text { for all } e \text { in } E, \\
x_{e} x_{f} & =0 & & \text { if }[e] \neq f \text { and } e, f \in E_{n}, \\
x_{e}^{*} x_{f} & =0 & & \text { if } e \neq f \text { and } e, f \in E_{n}, \\
x_{e} x_{f}^{*} & =0 & & \text { if }[e] \neq[f] \text { and } e, f \in E_{n}, \\
x_{e}^{*} x_{e} & =x_{f}^{*} x_{f} & & \text { if }[e]=[f] \text { and } e, f \in E_{n}, \\
x_{[e]} & =\left(x_{[e]}^{*} x_{[e]}\right)^{1 / 2} & & \text { for all } e, \\
x_{[e]} & \gg x_{f} x_{f}^{*} & & \text { if } e \in E_{n}, f \in E_{n+1} \text { and } r(e)=s(f) .
\end{aligned}
$$

For the proof we shall need some notations and some preliminary results. We use the graph-theoretic notation explained earlier, and let $S_{n}$ denote the set of paths in the Bratteli diagram from the root to the $n$th level. So each $\bar{e}$ in $S_{n}$ has the form

$$
\bar{e}=e_{1} e_{2} \ldots e_{n} \quad\left(e_{j} \in E_{j}\right) .
$$

The preferred paths of length $n$ are denoted

$$
\left[S_{n}\right]=\left\{\bar{e}=e_{1} e_{2} \ldots e_{n} \mid e_{j} \in\left[E_{j}\right]\right\} .
$$

For each path $\bar{e}$ in $S_{n}$ there is a unique path $[\bar{e}]$ in $\left[S_{n}\right]$ with $r([\bar{e}])=r(\bar{e})$.

We combine the edge-generators $x_{e}$ given in Theorem 6.4 to obtain the pathgenerators $x_{\bar{e}}$, defined by

$$
x_{\bar{e}}=x_{e_{1}} x_{e_{2}} \ldots x_{e_{n}}
$$

if $\bar{e}=e_{1} e_{2} \ldots e_{n}$.

6.5. Lemma. With notation as above, the elements $x_{\bar{e}}, \bar{e} \in \bigcup S_{n}=S$, satisfy the relations

$$
\begin{aligned}
\left\|x_{\bar{e}}\right\| & \leq 1 & & \text { for all } \bar{e} \text { in } S, \\
x_{\bar{e}} x_{\bar{f}} & =0 & & \text { if }[\bar{e}] \neq \bar{f} \text { and } \bar{e}, \bar{f} \in S_{n}, \\
x_{\bar{e}}^{*} x_{\bar{f}} & =0 & & \text { if } \bar{e} \neq \bar{f} \text { and } \bar{e}, \bar{f} \in S_{n}, \\
x_{\bar{e}} x_{\bar{f}}^{*} & =0 & & \text { if }[\bar{e}] \neq[\bar{f}] \text { and } \bar{e}, \bar{f} \in S_{n}, \\
x_{\bar{e}}^{*} x_{\bar{e}} & =x_{\bar{f}}^{*} x_{\bar{f}} & & \text { if }[\bar{e}]=[\bar{f}] \text { and } \bar{e}, \bar{f} \in S_{n}, \\
x_{[\bar{e}]} & =\left(x_{[\bar{e}}^{*} x_{[\bar{e}]}\right)^{1 / 2} & & \text { for all }[\bar{e}], \\
x_{[\bar{e}]} & \gg x_{\bar{f}} x_{\bar{f}}^{*} & & \text { if } \bar{e} \in S_{n}, \text { and } \bar{f}=\bar{e} \text { for some } f \text { in } E_{n+1} .
\end{aligned}
$$

Proof. For $n=1$ the conditions (i)-(vii) are given by definition. Assume they have been established for paths in $S_{1} \cup \cdots \cup S_{n}$ and consider $\bar{e}$ and $\bar{f}$ in $S_{n+1}$ of the form $\bar{e}=\widehat{e} e$ and $\bar{f}=\widehat{f} f$ with $e, f$ in $E_{n+1}$. Since $r(\widehat{e})=r([\widehat{e}])$, there is a path $[\widehat{e}] e$. Moreover, $[\bar{e}]=\widehat{d}[e]$ for some $\widehat{d}$ in $\left[S_{n}\right]$. Now compute

$$
x_{\bar{e}}^{*} x_{\bar{e}}=x_{e}^{*} x_{\hat{e}}^{*} x_{\hat{e}} x_{e}=x_{e}^{*} x_{[\hat{e}]}^{2} x_{e}=x_{e}^{*} x_{e}=x_{[e]}^{2},
$$

using (v)-(vii) for $S_{n}$. Moreover,

$$
x_{[\bar{e}]} x_{[\bar{e}]}^{*}=x_{\hat{d}} x_{[e]} x_{[e]}^{*} x_{\hat{d}}^{*}=x_{[e]}^{2}
$$

by (vi) and (vii), since $\widehat{d} \in\left[S_{n}\right]$. Combining the two computations we get (v), (vi) and (vii) for $S_{n+1}$. 
Using also the other path $\bar{f}$ we get

$$
x_{\bar{e}}^{*} x_{\bar{e}} x_{\bar{f}}=x_{[e]}^{2} x_{\hat{f}} x_{f}=x_{[e]}^{2} x_{\hat{d}} x_{\hat{f}} x_{f}=0
$$

if $\widehat{d} \neq \widehat{f}$ by (ii), using that $x_{\hat{d}} \gg x_{[e]}$. If, on the other hand $\widehat{d}=\widehat{f}$ (so $\widehat{f} \in\left[S_{n}\right]$ ), then

$$
x_{\bar{e}}^{*} x_{\bar{e}} x_{\bar{f}}=x_{[e]}^{2} x_{\hat{f}} x_{f}=x_{[e]}^{2} x_{f}=0
$$

unless $f=[e]$. Combining the computations we see that $x_{\bar{e}} x_{\bar{f}}=0$ unless $\bar{f}=[\bar{e}]$, which gives (ii) for $S_{n+1}$. To prove (iii),

$$
x_{\bar{e}}^{*} x_{\bar{f}}=x_{e}^{*} x_{\hat{e}}^{*} x_{\hat{f}} x_{f}=0
$$

unless $\widehat{e}=\widehat{f}$, in which case (by (vii))

$$
x_{\bar{e}}^{*} x_{\bar{f}}=x_{e}^{*} x_{f}=0
$$

if $e \neq f$ by definition. Finally,

$$
x_{\bar{e}}^{*} x_{\bar{e}} x_{\bar{f}}^{*} x_{\bar{f}}=x_{[e]}^{2} x_{[f]}^{2}=0
$$

unless $[e]=[f]$ by definition, in which case $r(e)=r(f)$ and so $[\bar{e}]=[\bar{f}]$. This proves (iv) for $S_{n+1}$, and the lemma follows by induction.

Proof of Theorem 6.4 . Let $B$ denote the universal $C^{*}$-algebra satisfying the relations (i)-(vii). It is elementary to check that these relations are also satisfied by the elements $\alpha_{n} \otimes v_{q(e), p(e)}$ defined previously, and since these generate the telescope $\mathbf{T}(A)$, cf. Lemma 6.3, it follows that the assignment

$$
x_{e} \mapsto \alpha_{n} \otimes v_{q(e), p(e)}
$$

gives a surjective morphism of $B$ onto $\mathbf{T}(A)$.

To construct the inverse morphism note that the relations (i)-(vi) in Lemma 6.5 show that the assignment

$$
\operatorname{id}^{2} \otimes v_{\bar{e}, \bar{f}} \mapsto x_{\bar{e}} x_{\bar{f}}^{*},
$$

where $\bar{e}, \bar{f} \in S_{n}$, and $r(\bar{e})=r(\bar{f})$, induces a ${ }^{*}$-homomorphism $\varphi_{n}: \mathbf{C} A_{n} \rightarrow B$. Indeed,

$$
\varphi_{n}\left(\gamma\left(t^{2}\right) t^{2} v_{\bar{e}, \bar{f}}\right)=x_{\bar{e}} \gamma\left(x_{[e]}^{2}\right) x_{\bar{f}}^{*}
$$

for each $\gamma$ in $\left.\left.C_{0}(] 0,1\right]\right)$ (cf. the proof of Lemma 6.1), so we know $\varphi_{n}$ on a set whose closed linear span is $\mathbf{C} A_{n}$.

For $m<n$, if $\bar{e}, \bar{f} \in S_{m}$ and $\bar{g}, \bar{h} \in S_{n}$, then

$$
\varphi_{m}\left(\gamma\left(t^{2}\right) t^{2} v_{\bar{e}, \bar{f}}\right) \varphi_{n}\left(\beta\left(t^{2}\right) t^{2} v_{\bar{g}, \bar{h}}\right)=x_{\bar{e}} \gamma\left(x_{[e]}^{2}\right) x_{\bar{f}}^{*} x_{\bar{g}} \beta\left(x_{[g]}^{2}\right) x_{\bar{h}}^{*}=0,
$$

by (iii), unless $\bar{g}=\overline{f d}$ for some path $\bar{d}$, in which case the product above becomes

$$
x_{\bar{e}} \alpha\left(x_{[\bar{e}]}^{2}\right) x_{[\bar{f}]}^{2} x_{\bar{d}} \beta\left(x_{[\bar{g}]}^{2}\right) x_{\bar{h}}^{*} .
$$

Note that $[\bar{e}]=[\bar{f}]$ and $[\bar{g}]=[\bar{h}]$, since they have the same ranges. Note also that $x_{[e]} x_{\bar{d}}=x_{\bar{d}}$, since $s(\bar{d})=r(\bar{f})(=r(\bar{e}))$ by (vii), so that the product above becomes

$$
\begin{aligned}
x_{\bar{e}} \gamma(1) x_{\bar{d}} \beta\left(x_{[\bar{h}]}^{2}\right) x_{\bar{h}}^{*} & =x_{\bar{e} \bar{d}} \gamma(1) \beta\left(x_{[\bar{h}]}^{2}\right) x_{\bar{h}}^{*} \\
& =\varphi_{n}\left(\gamma(1) \beta\left(t^{2}\right) t^{2} v_{\bar{e} \bar{d}, \bar{h}}\right) \\
& =\varphi_{n}\left(\gamma(1) v_{\bar{e}, \bar{f}} \beta\left(t^{2}\right) t^{2} v_{\bar{g}, \bar{h}} .\right.
\end{aligned}
$$


We have shown that, for $a$ in $\mathbf{C} A_{m}$ and $b$ in $\mathbf{C} A_{n}$,

$$
\varphi_{m}(a) \varphi_{n}(b)=\varphi_{n}(a(1) b)
$$

where $a(1) b$ describes the action of $\mathbf{C} A_{m}$ in $M\left(\mathbf{C} A_{n}\right)$. By Corollary 2.5 such a coherent sequence of morphisms $\left(\varphi_{n}\right)$ determines a unique morphism $\varphi$ of $\mathbf{T}(A)$ into $B$.

Finally, since $\left.\varphi\right|_{\mathbf{C} A_{n}}=\varphi_{n}$ only after identifying $\left.] n-1, n\right]$ with $\left.] 0,1\right]$, we get

$$
\varphi\left(\alpha_{n}^{2} \otimes v_{q(e), p(e)}\right)=1 x_{q(e)} x_{p(e)}^{*} .
$$

Here $q(e)=e_{1} e_{2} \ldots e_{n-1} e$, where $e_{k} \in[E]$ for $1 \leq k \leq n-1$, whereas $p(e)=$ $f_{1} f_{2} \ldots f_{n}$ with $f_{k}$ in $[E]$ for all $k$. Thus

$$
\varphi\left(\alpha_{n} \otimes v_{q(e), p(e)}\right)=x_{e_{1}} x_{e_{2}} \ldots x_{e_{n-1}} x_{e} x_{f_{n}} \ldots x_{f_{1}}=x_{e},
$$

because the $x_{e_{k}}$ 's and the $x_{f_{k}}$ 's act as units against $x_{e}$ by conditions (v)-(vii) in Theorem 6.4. This proves that $\varphi$ is indeed the inverse of our first morphism of $B$ onto $\mathbf{T}(A)$, and thus an isomorphism.

To appreciate the presentation in Theorem 6.4 note the economy: The blunt approach via matrix units would give a set of generators $\alpha_{n} \otimes v_{\bar{e}, \bar{f}}$, labeled by all possible paths in $\bigcup S_{n}$. In our presentation the generators are labeled by the edges only - taking advantage of the structure of the previous subalgebras. To illustrate the effect we offer the following simple example.

6.6. Proposition. Let $\widetilde{K}$ denote the unitized $C^{*}$-algebra of compact operators on the Hilbert space $\ell^{2}$, realized as the inductive limit of matrix algebras $\mathbb{M}_{n} \oplus \mathbb{C}$, as usual. Then the mapping telescope $\mathbf{T}(\widetilde{K})$ has a presentation with generators $\left(x_{n}\right)$ and $\left(h_{n}\right)$, subject to the relations

$$
\begin{aligned}
\left\|x_{n}\right\| & \leq 1, \\
0 & \leq h_{n} \leq 1, \\
x_{1} h_{1} & =h_{1} x_{1}=0, \\
0 & \leq x_{1}, \\
x_{n}^{2} & =h_{n} x_{n}=0 \quad(n \geq 2), \\
x_{n}^{*} x_{n} & \gg x_{n+1}^{*} x_{n+1}, \\
h_{n} & \gg x_{n+1} x_{n+1}^{*}, \\
h_{n} & \gg h_{n+1} .
\end{aligned}
$$

Proof. With $\alpha_{n}(t)=((t+1-n) \vee 0) \wedge 1$ as before, and with $e_{i j}$ the usual matrix units in $\mathbb{B}\left(\ell^{2}\right)$, we define our generators by

$$
\begin{aligned}
& x_{n}=\alpha_{n} \otimes e_{n 1}, \\
& h_{n}=\alpha_{n} \otimes \sum_{k=n+1}^{\infty} e_{k k} .
\end{aligned}
$$

That these elements satisfy the relations and generate $\mathbf{T}(\widetilde{K})$ is immediate. 
The Bratteli diagram for $\widetilde{K}$ is

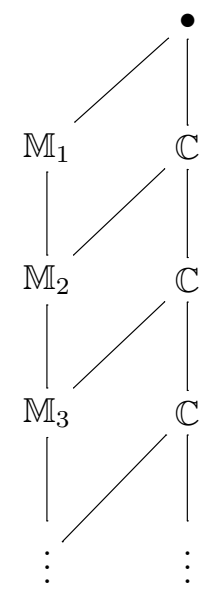

so our model - labeling the generators by the edges - should really contain a third sequence of generators $\left(y_{n}\right)$. These are the redundant ones (cf. Remark 6.2) given by

$$
y_{n}=\alpha_{n} \otimes e_{n n}=\left(x_{n}^{*} x_{n}\right)^{1 / 2} .
$$

6.7. Remark. In Theorem 6.4, if the generators (and accordingly the relations) are restricted to

$$
\left\{x_{e} \mid e \in E_{1} \cup \cdots \cup E_{n}\right\},
$$

then the universal $C^{*}$-algebra is

$$
\mathbf{T}\left(A_{1}, \ldots, A_{n}\right)
$$

If we add the relations

$$
x_{[e]}^{2}=x_{[e]} \quad\left(e \in E_{j}\right)
$$

for all $j$, then the presentation becomes one for the AF algebra $A=\overline{\bigcup A_{n}}$, or if the generators are truncated, for $A_{n}$.

6.8. Remark. Let $B_{n}$ be the subalgebra of $A_{n}$ generated, in the path model for $A_{n}$, by matrix units corresponding to pairs of paths that have first edge in $\left[E_{1}\right]$. Notice that $B_{1}$ is commutative, and $B_{n} \subseteq B_{n+1}$. Let $B=\overline{\cup B_{n}}$. If in Theorem 6.4 the generators

$$
\left\{x_{e} \mid e \in E_{1} \backslash\left[E_{1}\right]\right\}
$$

are omitted, the resulting $C^{*}$-algebra is

$$
\mathbf{T}(B)=\mathbf{T}\left(B_{1}, B_{2}, \ldots\right) .
$$

Since there are no relations between the dropped generators and the $x_{f}$ for $f \in E_{n}$ when $n \geq 2$, we immediately obtain the isomorphism

$$
\mathbf{T}\left(A_{1}, A_{2}, \ldots\right) \cong \mathbf{C} A_{1} *_{\mathbf{C} B_{1}} \mathbf{T}\left(B_{1}, B_{2}, \ldots\right) .
$$

Similarly

$$
A \cong A_{1} *_{B_{1}} B
$$


which generalizes the well-known isomorphism

$$
\mathbf{M}_{n}(B) \cong \mathbb{M}_{n} *_{\mathbb{C}} B
$$

(amalgamating so that $e_{11} \leftrightarrow 1_{B}$ ), which holds for all unital $\mathbb{C}$-algebras, not just for unital AF algebras.

\section{Projectivity of AF-Telescopes}

As in the previous section, we consider a fixed unital Bratteli diagram (with root and multiple edges) corresponding to a system of finite-dimensional $C^{*}$-algebras

$$
\mathbb{C} \subseteq A_{1} \subseteq A_{2} \subseteq \ldots,
$$

and we put $A=\bar{\bigcup} A_{n}$. We denote by $E=\bigcup E_{n}$ the set of edges in the diagram and define $e \sim f$ if $r(e)=r(f)$ (for $e, f$ in some $E_{n}$ ). Then we choose a set $[E]=\bigcup\left[E_{n}\right]$ of preferred edges, one from each equivalence class, and let $e \mapsto[e]$ denote the selection function. It follows from Theorem 6.4 that each of the finite telescopes $\mathbf{T}\left(A_{1}, A_{2}, \ldots, A_{n+1}\right)$ is the universal $C^{*}$-algebra generated by elements $x_{e}, e \in \bigcup_{k=1}^{n} E_{k}$, subject to the relations (i)-(vii) in that theorem.

To facilitate the lifting process we shall need the additional elements $h_{e}, e \in\left[E_{n}\right]$, defined by $h_{e}=\sum x_{f} x_{f}^{*}$, the summation being over all $f$ in $E_{n+1}$ with $s(f)=r(e)$. Thus $h_{e} \ll x_{e}$ for every $e$ in $\left[E_{n}\right]$ by condition (vii). These additional elements come free, as we see from the next lemma.

7.1. Lemma. With notation as in Lemma 6.1, let

$$
F=\bigoplus_{[S]} \mathbb{M}_{\#[e]} .
$$

Let (i)-(vi) denote the relations (i)-(vi) given in Lemma 6.1 in the variable $\left\{x_{e} \mid\right.$ $e \in S\}$. With the additional variables $\left\{h_{e} \mid e \in[S]\right\}$ consider the relations

$$
\begin{aligned}
& 0 \leq h_{e} \leq 1, \\
& h_{e} \ll x_{e} \quad(e \in[S]) .
\end{aligned}
$$

(1) The universal $C^{*}$-algebra generated by the $x_{e}$ and the $h_{[e]}$ subject to (i)-(viii) is $\mathbf{T}(F, F) \cong \mathbf{C} F$.

(2) Suppose $x_{e}$ and $h_{[e]}$ (for all $e \in S$ ) are elements of some quotient $B / J$ of a $C^{*}$-algebra $B$ satisfying (i)-(viii). Suppose further that for each $e \in S$ there is a hereditary subalgebra $B_{e}$ of $B$ with $x_{e} \in \pi\left(B_{e}\right)$ and that

$$
e \neq f \Longrightarrow\left(B_{e}=B_{f} \text { or } B_{e} \perp B_{f}\right) .
$$

Then there exist $y_{e}$ and $k_{[e]}$ in $B$ with $\pi\left(y_{e}\right)=x_{e}$ and $\pi\left(k_{e}\right)=h_{e}$ that satisfy (i)-(viii) and so that $y_{e} \in B_{e}$ for all $e$.

Proof. Evidently part (1) follows from Remark 6.7. If $\alpha$ and $\beta$ are the functions on $[0,2]$ given by $\alpha(t)=t \wedge 1, \beta(t)=(t-1) \vee 0$, then a specific set of generators for $\mathbf{T}(F, F)$ are

$$
\begin{aligned}
& x_{e}=\alpha \otimes v_{e,[e]} \quad(e \in S), \\
& h_{e}=\beta \otimes v_{e, e} \quad(e \in[S]) .
\end{aligned}
$$

Notice that $\alpha \otimes v_{e, e}$ and id $\otimes v_{e, e}$ generate the same hereditary subalgebra of $\mathbf{C} F$ (namely $\mathbf{C}\left(\mathbb{C} v_{e e}\right)$ ), and so (2) is equivalent to Theorem 3.7. 
7.2. Theorem. The mapping telescope of every countable inductive limit of finitedimensional $C^{*}$-algebras $\left(A_{n}\right)$ is projective.

Proof. Assume first that the inductive limit is unital and put $A=\overline{\bigcup A_{n}}$. Then, with notation as above, consider the set $\left\{x_{e} \mid e \in E\right\}$ of generators for $\mathbf{T}(A)$.

Given a quotient map $\pi: B \rightarrow Q$ between $C^{*}$-algebras and a morphism $\varphi$ : $\mathbf{T}(A) \rightarrow Q$, we may assume, working by induction, that for some $n$ we have found elements

$$
\left\{y_{e} \mid e \in \bigcup_{k=1}^{n-1} E_{k}\right\} \quad \text { and } \quad\left\{k_{e} \mid e \in\left[E_{n-1}\right]\right\}
$$

in $B$, such that $\pi\left(y_{e}\right)=\varphi\left(x_{e}\right), \pi\left(k_{e}\right)=\varphi\left(h_{e}\right)$, and such that the $y_{e}$ 's satisfy the relations (i)-(vii) in Theorem 6.4 , whereas $k_{e} \ll y_{e}$ for every $e$ in $\left[E_{n-1}\right]$. Note that for $n=1$ no choices have been made.

For each $e$ in $\left[E_{n-1}\right]$ let $B_{e}$ denote the hereditary $C^{*}$-subalgebra of $B$ generated by $k_{e}$. Since $\pi\left(k_{e}\right)=h_{e}$, it follows that $\varphi\left(x_{f} x_{f}^{*}\right) \in \pi\left(B_{e}\right)$ for every $f$ in $E_{n}$ with $s(f)=r(e)$. We can therefore apply Theorem 3.7 to find $y_{f}$ and $k_{g}\left(g \in\left[E_{n}\right]\right)$ such that (i)-(viii) hold, $k_{g} \ll y_{g}$ and $y_{f} y_{f}^{*} \in B_{e}$ if $s(f)=r(e)$. The crucial condition (vii) follows because $y_{f} y_{f}^{*} \in B_{e}$, and $y_{e}$ is a unit for $B_{e}$, because $k_{e} \ll y_{e}, e \in\left[E_{n-1}\right]$.

Continuing by induction, we obtain a sequence $\left\{y_{e} \mid e \in E\right\}$ that satisfies the conditions (i)-(vii) in Theorem 6.4. (The additional elements $\left\{k_{e} \mid e \in[E]\right\}$ we discard.) By universality this defines a morphism $\psi: \mathbf{T}(A) \rightarrow B$; and since $\pi\left(y_{e}\right)=$ $\varphi\left(x_{e}\right)$ for every $e$ in $E$, it follows that $\pi \circ \psi=\varphi$, whence $\mathbf{T}(A)$ is projective.

The condition that all embeddings are unital is removed by Propositions 5.4, so we conclude that $\mathbf{T}(A)$ is projective for any sequence $\left(A_{n}\right)$ of finite-dimensional $C^{*}$-algebras.

\section{REFERENCES}

1. C. A. Akemann and G. K. Pedersen, Ideal perturbations of elements in $C^{*}$-algebras, Math. Scand. 41 (1977), 117-139. MR 57:13507

2. B. Blackadar, Shape theory for $C^{*}$-algebras, Math. Scand. 56 (1985), 249-275. MR 87b:46074

3. B. Blackadar and E. Kirchberg, Generalized inductive limits of finite-dimensional $C^{*}$-algebras, Math. Ann. 307 (1997), 343-380. MR 98c:46112

4. O. Bratteli, Inductive limits of finite dimensional $C^{*}$-algebras, Trans. Amer. Math. Soc. 171 (1972), 195-234. MR 47:844

5. R. C. Busby, Double centralizers and extensions of $C^{*}$-algebras, Trans. Amer. Math. Soc. 132 (1968), 79-99. MR 37:770

6. F. Combes, Sur les faces d'une $C^{*}$-algèbre, Bull. Sci. Math. 93 (1969), 37-62. MR 42:856

7. J. Cuntz, Simple $C^{*}$-algebras generated by isometries, Commun. Math. Phys. 57 (1977), 173-185. MR 57:7189

8. K. R. Davidson, Lifting positive elements in $C^{*}$-algebras, Integral Eq. and Operator Theory 14 (1991), 183-191. MR 92f:46065

9. E. G. Effros and J. Kaminker, Homotopy, continuity and shape theory for $C^{*}$-algebras, Geometric Methods in Operator Algebras, Editors H. Araki \& E. G. Effros, Pitman Res. Notes 123 (1986), 152-180. MR 88a:46082

10. R. Exel and T. A. Loring, Finite-dimensional representations of free product $C^{*}$-algebras, Int. J. Math. 3 (1992), 469-476. MR 93f:46091

11. J. Glimm, On a certain class of operator algebras, Trans. Amer. Math. Soc. 95 (1960), 318340. MR 22:2915

12. J. Glimm, Type I $C^{*}$-algebras, Annals of Math. 73 (1961), 572-612. MR 23:A2006

13. J. Glimm and R. V. Kadison, Unitary operators in $C^{*}$-algebras, Pacific J. Math. 10 (1960), 547-556. MR 22:5906 
14. K. R. Goodearl and P. Menal, Free and residually finite-dimensional $C^{*}$-algebras, J. Funct. Anal. 90 (1990), 391-410. MR 91f:46078

15. R. V. Kadison, Irreducible operator algebras, Proc. Natl. Acad. Sci. USA 43 (1957), 273-276. MR 19:47e

16. T. A. Loring, $C^{*}$-algebras generated by stable relations, J. Funct. Anal. 112 (1993), 159-201. MR 94k:46115

17. T. A. Loring, Projective $C^{*}$-algebras, Math. Scand. 73 (1993), 274-280. MR 95h:46085

18. T. A. Loring, Stable relations II: corona semiprojectivity and dimension-drop $C^{*}$-algebras, Pacific J. Math. 172 (1996), 461-475. MR 97c:46070

19. C. L. Olsen and G. K. Pedersen, Corona $C^{*}$-algebras and their applications to lifting problems, Math. Scand. 64 (1989), 63-86. MR 91g:46064

20. G. K. Pedersen, Isomorphisms of UHF algebras, J. Funct. Anal. 30 (1978), 1-16. MR 80m:46055

21. G. K. Pedersen, $C^{*}$-Algebras and their Automorphism Groups, LMS Monographs 14, Academic Press, London/New York, 1979. MR 81e:46037

22. G. K. Pedersen, $S A W^{*}$-algebras and corona $C^{*}$-algebras. Contributions to non-commutative topology, J. Operator Theory 15 (1986), 15-32. MR 87a:46095

23. G. K. Pedersen, The corona construction, Proc. of the 1988 GPOTS-Wabash Conf., Editors J. B. Conway and B. B. Morrel, Pitman Res. Notes 225 (1990), 49-92. MR 92e:46119

24. C. Schochet, Topological methods for $C^{*}$-algebras III: axiomatic homology, Pacific J. Math. 114 (1984), 399-445. MR 86g:46102

25. N. E. Wegge-Olsen, K-Theory and $C^{*}$-Algebras, Oxford Univ. Press, Oxford 1993. MR 95c: 46116

Department of Mathematics, University of New Mexico, Albuquerque, New Mexico 87131

E-mail address: loring@math.unm.edu

Mathematics Institute, University of Copenhagen, Universitetsparken 5, DK-2100 Copenhagen $\varnothing$, Denmark

E-mail address: gkped@math.ku.dk 